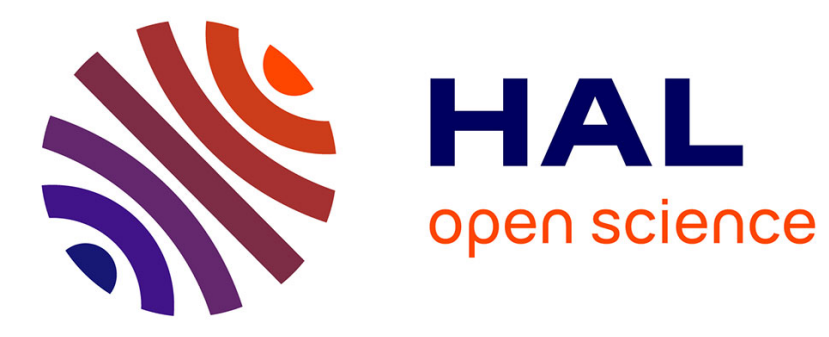

\title{
Non-linear analysis of a model for yeast cell communication
}

Vincent Calvez, Thomas Lepoutre, Nicolas Meunier, Nicolas Muller

\section{To cite this version:}

Vincent Calvez, Thomas Lepoutre, Nicolas Meunier, Nicolas Muller. Non-linear analysis of a model for yeast cell communication. ESAIM: Mathematical Modelling and Numerical Analysis, 2020, 54 (2), pp.619 - 648. 10.1051/m2an/2019065 . hal-02372511

\section{HAL Id: hal-02372511 \\ https://hal.inria.fr/hal-02372511}

Submitted on 20 Nov 2019

HAL is a multi-disciplinary open access archive for the deposit and dissemination of scientific research documents, whether they are published or not. The documents may come from teaching and research institutions in France or abroad, or from public or private research centers.
L'archive ouverte pluridisciplinaire HAL, est destinée au dépôt et à la diffusion de documents scientifiques de niveau recherche, publiés ou non, émanant des établissements d'enseignement et de recherche français ou étrangers, des laboratoires publics ou privés. 


\title{
NON-LINEAR ANALYSIS OF A MODEL FOR YEAST CELL COMMUNICATION
}

\author{
Vincent Calvez ${ }^{1}$, Thomas Lepoutre ${ }^{2}$, Nicolas Meunier $^{3}$ and Nicolas Muller
}

\begin{abstract}
We study the non-linear stability of a coupled system of two non-linear transport-diffusion equations set in two opposite half-lines. This system describes some aspects of yeast pairwise cellular communication, through the concentration of some protein in the cell bulk and at the cell boundary. We show that it is of bistable type, provided that the intensity of active molecular transport is large enough. We prove the non-linear stability of the most concentrated steady state, for large initial data, by entropy and comparison techniques. For small initial data we prove the self-similar decay of the molecular concentration towards zero. Informally speaking, the rise of a dialog between yeast cells requires enough active molecular transport in this model. Besides, if the cells do not invest enough in the communication with their partner, they do not respond to each other; but a sufficient initial input from each cell in the dialog leads to the establishment of a stable activated state in both cells.
\end{abstract}

Résumé. Nous étudions la stabilité non linéaire d'un système couplé de deux équations de transportdiffusion non linéaires définies sur des demi-droites. Ce système décrit certains aspects de la communication cellulaire entre levures, par la concentration de certaines protéines dans et sur la membrane de la cellule. Nous montrons qu'il est de type bistable, pour un taux de transport actif suffisamment important. Nous prouvons la stabilité non linéaire de l'état d'équilibre le plus concentré, via l'entropie et des techniques de comparaison. Pour des données initiales petites, nous prouvons la décroissance auto-similaire de la concentration vers zéro. De manière informelle, la montée d'un dialogue entre cellules nécessite suffisamment de transport moléculaire actif dans ce modèle. En outre, si les cellules n'investissent pas suffisamment dans la communication avec leur partenaire, elles ne se répondent pas; mais un dialogue initialement suffisamment initié conduit à l'établissement d'un état activé stable dans les deux cellules.

1991 Mathematics Subject Classification. 35B32, 35B35, 35B40, 35K40, 35Q92, 92B05, 92C17, 92C37.

\section{INTRODUCTION, MODEL AND RESULTS}

How do cells communicate with each other? This question, which seems simple, is still under current investigation. Cell communication plays fundamental role in many cellular processes including cell division and differentiation, directional movement as well as morphogenesis. Defects in cell-cell communication are also involved in the development of cancer.

Keywords and phrases: Non-linear stability, asymptotic convergence,logarithmic Sobolev inequality, HWI inequality

${ }^{1}$ Univ Lyon, CNRS, Université Claude Bernard Lyon 1, UMR5208, Institut Camille Jordan, F-69622 Villeurbanne, France

2 Univ Lyon, Inria, Université Claude Bernard Lyon 1, CNRS UMR5208, Institut Camille Jordan, F-69603 Villeurbanne, France

${ }^{3}$ LaMME, UMR 8071 CNRS \& Université Évry Val d'Essonne, France 
From the biological point of view, a prototypical model for cell communication is given by yeast cell mating. In this work we propose and analyze a minimal model describing some aspects of yeast cell-cell communication. This model is based on a system of coupled non-linear and transport-diffusion equations. The uncoupled transport-diffusion equation, was introduced and studied in previous works to describe internal protein dynamics in a single yeast cell $8,9,21,28,32$, including the process of spontaneous polarization. Here, from the mathematical viewpoint, the novelty is the coupling between two such equations set on two opposite half-lines. The coupling occurs from some scalar quantities which account for molecular communication between cells.

In the context of yeast cell communication, the main interest of this model is to link the output of cell communication to protein aggregation on both cell membranes. Indeed, the capacity of a cell to interact with other cells depends on its intracellular protein distribution $n_{i}$ : proteins located on the cell membrane $\left(\mu_{i}\right)$ are able to combine with some exterior signalling pheromone to enhance active transport of proteins to produce some signal that will diffuse in the exterior medium. Hence, in a cell where the proteins are located in its bulk, the communication with the environment will be very difficult. On the contrary, a cell with many proteins on its membrane will have a great capacity for communication.

A one-dimensional model for cell-cell communication. In the following, each cell $i=1,2$ is modelled as a halfline, with a boundary reduced to a single point (see Figure 1). As the coupling between the two cells occurs through a scalar quantity, there is no need to consider disjoint half-lines. So we consider that each cell coincides with $\mathbb{R}_{+}$, without loss of generality. Each cell is characterized by the distribution of some protein involved in the polarization process. The proteins can be in two states: either in the cell bulk - with density $n_{i}(t, x)-$ or attached to the cell boundary - with fraction $\mu_{i}(t)$. Accordingly, the total molecular content in each cell is:

$$
M_{i}(t):=\mu_{i}(t)+\int_{0}^{\infty} n_{i}(t, x) \mathrm{d} x
$$

The model consists of the following equations:

$$
\partial_{t} n_{i}(t, x)=\partial_{x x} n_{i}(t, x)+\chi \mu_{1}(t) \mu_{2}(t) \partial_{x} n_{i}(t, x), \quad t \geq 0, \quad x \geq 0
$$

for $i=1,2$, where $n_{i}=n_{i}(t, x)$ is the density of some molecular content in each cell bulk, $\mu_{i}(t)(i=1,2)$ is the fraction of molecules attached to each cell boundary at $x=0$, and $\chi>0$ is a parameter, which accounts for the strength of the coupling between the two cells. This parameter combines the effects of intracellular protein transport, as well as the level of interaction between the two cells that may depend, e.g. upon the mutual distance. The dynamics of $\mu_{i}(t)$ are given by attachment and detachment kinetic at the boundaries:

$$
\frac{\mathrm{d} \mu_{i}}{\mathrm{~d} t}(t)=n_{i}(t, 0)-\mu_{i}(t), \quad t>0
$$

Moreover, equations (2) are complemented with the following flux boundary conditions:

$$
\frac{\mathrm{d} \mu_{i}}{\mathrm{~d} t}(t)=\partial_{x} n_{i}(t, 0)+\chi \mu_{1}(t) \mu_{2}(t) n_{i}(t, 0), \quad t>0
$$

which ensure the conservation of the total molecular content in each cell $M_{i}(t)=M_{i}(0)$ (1).

Briefly, the main assumptions that lead to (2) are the following: (i) Proteins in the bulk can be either freely diffusing or actively transported towards the cell boundary, (ii) The effects of activated proteins attached to the boundary $\left(\mu_{i}\right)$ are twofolds as they enhance active transportation in both cells. The underlying processes are: nucleation of actin filaments in one cell, and secretion of some external signalling molecule (pheromone) which can bind to the other cell. We refer to Section 2 for a detailed presentation of the model with biological motivations. 


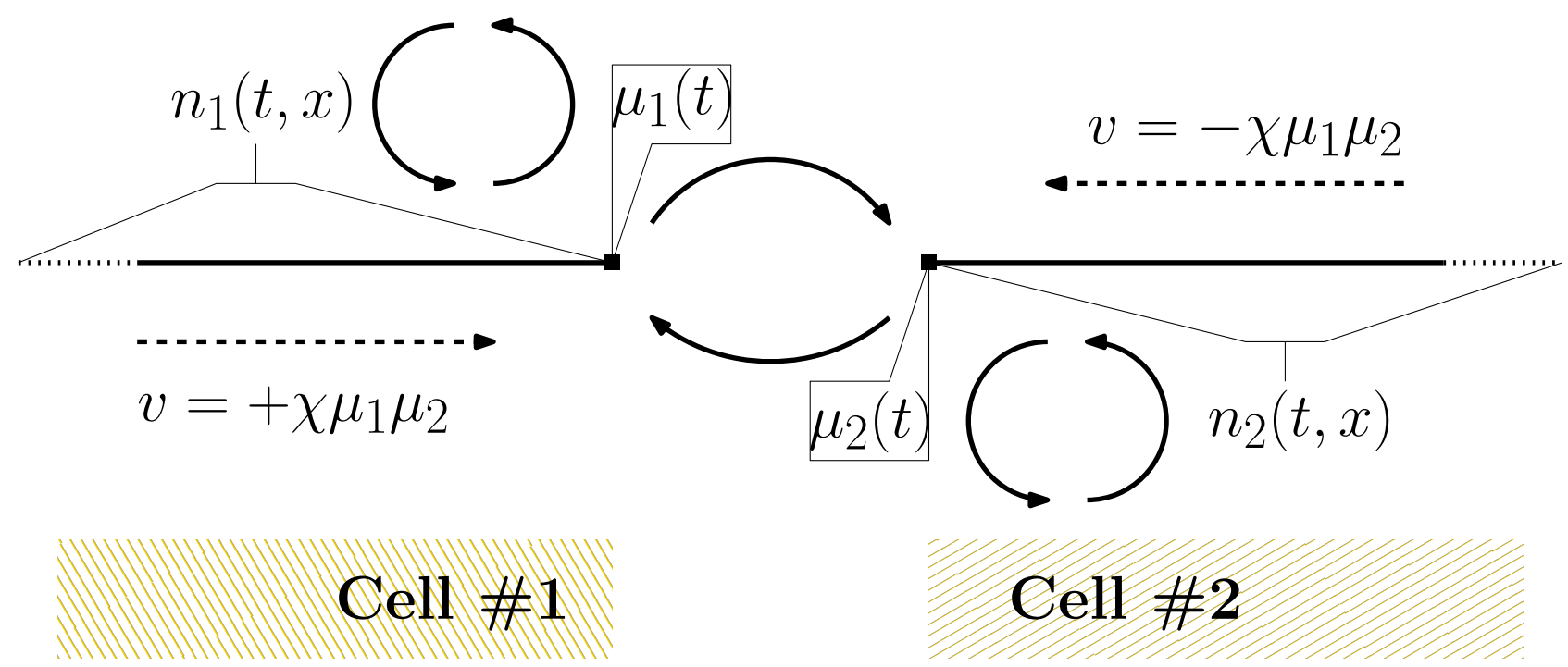

Figure 1. Cartoon of the model (2)-(3)-(4): the molecular content in the bulk experiences diffusion and transport at speed $v= \pm \chi \mu_{1} \mu_{2}$, where $\mu_{i}$ can be seen as the fraction of activated molecules attached to the cell boundary. The dialog between the two cells arises as they secrete some communication signal (a pheromone) at a rate proportional to $\mu_{i}$, enhancing further the transport of molecular content in the opposite cell. Details are given in Section 2.

For the sake of simplicity, throughout this work we assume that both cells have the same (conserved) total molecular content which we normalize as follows:

$$
M_{1}(t)=M_{2}(t)=1 .
$$

Objectives and state of the art. Since the transport speed is bounded, $\mu_{i}(t) \leq 1$, global existence of solutions to the Cauchy problem (2)-(3)-(4) holds true. We refer to Appendix C for the sketch of the well-posedness theory for the PDE-ODE coupled system. Here, our aim is to precise the long time behaviour. In order to bypass the lack of comparison principle on equation (2), our method is based on a concentration-comparison principle that is obtained when equation (2) is integrated in space, see [28. This principle allows constructing some remarkable sub/supersolutions and performing a non-linear stability analysis.

Before stating our results about the analysis of (2)-(3)-(4), let us briefly comment the literature. The uncoupled problem, corresponding to a single cell $(i=1$, say $)$ in a constant field of pheromone concentration $\left(\mu_{2} \equiv 1\right.$, say) was introduced in $[9$ and $[8]$ as the one-dimensional version of a class of active transport models introduced previously in 21] for the modelling of cell polarization. Two cases were considered: either a direct coupling $\mu_{i}(t)=n_{i}(t, 0)$, or a coupling via exchange of molecular content at the boundary, as (3). In the former case, the solution may blow up in finite time for large $\chi$, due to the strong interaction. In the latter case, the solution converges to a bounded stationary state for large $\chi$, but decays to zero for small $\chi$. The results were improved later in 28 using suitable comparison principles.

This dichotomy between concentration of the solution vs. self-similar decay is analogous to the classical Keller-Segel equation for chemotaxis [5]. However, the interaction here goes through the trace value at the boundary $n_{i}(t, 0)$, which makes the analysis more difficult. It is more singular, and furthermore it lacks symmetry properties and in particular there is no free energy associated with (2)-(3)-(4) to our knowledge. Nevertheless, we show here that the system inherits some structure from this analogy. In particular, it is possible to construct Lyapunov functionals that contain some entropy contributions. This is quite remarkable as the system is not linear. 
Finally, let us mention that similar models involving a coupling between a one-dimensional PDE and a scalar boundary value appear in the modelling of NNLIF models 6, 7, 10, 11, 16], except that the derivative at the boundary is involved, among other differences. Nevertheless, entropy techniques have also been used in this context, as well as moment estimates.

All the aforementioned works deal with only one density (of proteins or attracting cells or firing neurons). In the present work we show that many results can be extended to the nonlinear coupling between two protein densities, as in Figure 1. Besides, the underlying cubic nonlinearity yields a bistable behaviour which deserves some careful analysis.

Notations. Let us fix some notations. For $i=1,2$, the function $N_{i}$ is defined as the cumulated distribution function of $\mu_{i}(t) \delta_{0}+n_{i}(t, x) \mathrm{d} x$ :

$$
N_{i}(t, x)=\mu_{i}(t)+\int_{0}^{x} n_{i}(t, y) \mathrm{d} y .
$$

Besides, for any parameter $\mu \in(0,1)$ we introduce the cumulated function $N_{\mu}$ associated with some prescribed value of $\mu_{1}=\mu_{2}=\mu$, which is defined by

$$
N_{\mu}(x)=\mu+(1-\mu)\left(1-e^{-\chi \mu^{2} x}\right)
$$

Such a distribution is associated to a stationary state of equation (2)-(3) -4 if we have the additional constraint $1-\mu=\frac{1}{\chi \mu}$. Suppose that $\chi \geq 4$. Then the polynomial $P$ defined by

$$
P(X)=\chi X^{2}-\chi X+1
$$

admits two real roots denoted by $\mu_{-} \leq \mu_{+}$. The stationary states of the coupled system $n_{+}$and $n_{-}$are defined, together with $m_{+}$and $m_{-}$, as follows:

$$
n_{ \pm}(x)=\left(1-\mu_{ \pm}\right) m_{ \pm}(x)=\left(1-\mu_{ \pm}\right) \chi \mu_{ \pm}^{2} e^{-\chi \mu_{ \pm}^{2} x}
$$

Statement of the main results. As the coupling goes through a cubic nonlinearity in the transport term of (2), it is expected that for small initial data, or alternatively for small parameter $\chi$, the system behaves essentially linearly with a diffusive behaviour. Indeed, we identified the threshold $\chi \lessgtr 4$ above which the system admits non-trivial steady states.

Proposition 1.1 (Steady states and linear stability). Consider the system (2)-(3) - (4) and (5). Any steady state $\left(\bar{n}_{i}, \bar{\mu}_{i}\right)$ satisfies $\bar{n}_{1}=\bar{n}_{2}$ and $\bar{\mu}_{1}=\bar{\mu}_{2}$. The following alternative holds true:

- If $\chi<4$, then the system does not admit a steady state.

- If $\chi>4$, then, there are two steady states $\left(n_{-}, \mu_{-}\right)$and $\left(n_{+}, \mu_{+}\right)$. Furthermore, $\left(n_{+}, \mu_{+}\right)$is linearly stable while $\left(n_{-}, \mu_{-}\right)$is linearly unstable.

- If $\chi=4$, then $\mu_{-}=\mu_{+}=1 / 2$ and the sytem admits a unique steady state.

Intuitively, the system is of bistable type, with a bifurcation at $\chi=4$. For $\chi<4$, the density is expected to converge to zero for any initial condition and to follow the self similar behaviour driven by the diffusion part. For $\chi>4$ it is expected either to follow the latter self similar behaviour or to converge to the most concentrated steady state $\left(n_{+}, \mu_{+}\right)$defined by (7), (8), depending on the initial data (see Table 1).

In this work we are interested in making this informal statement rigorous in the non-linear regime. Our main result is the convergence towards the steady state $\left(n_{+}, \mu_{+}\right)$defined by (7), (8), for large enough initial data.

Theorem 1.2 (Non-linear stability of the largest equilibrium). Assume $\chi>4$ and let $\left(n_{ \pm}, \mu_{ \pm}\right)$be defined by (7), (8). Let $\left(n_{i}, \mu_{i}\right)_{1 \leq i \leq 2}$ be the solution to (2) - (3) - (4) with initial data $\left(n_{i}^{0}, \mu_{i}^{0}\right)_{1 \leq i \leq 2}$ satisfying (5). Suppose that both the entropy and the first moment of the initial data $\left(n_{i}^{0}, \mu_{i}^{0}\right)_{1 \leq i \leq 2}$ are finite: $\int_{0}^{\infty} n_{i}^{0}(x)\left(x+\log n_{i}^{0}(x)\right) \mathrm{d} x<$ $+\infty$. Assume in addition that there exist two real numbers $\left(\underline{\mu}_{0}, \bar{\mu}_{0}\right) \in(0,1)^{2}$ ordered as follows,

$$
\mu_{-}<\underline{\mu}_{0}<\mu_{+}<\bar{\mu}_{0},
$$


such that the initial data are ordered as follows:

$$
(\forall x \geq 0) \quad N_{\underline{\mu}_{0}}(x) \leq N_{i}^{0}(x) \leq N_{\bar{\mu}_{0}}(x), \quad i=1,2 .
$$

Then, for $i=1,2$, the convergence of $\left(n_{i}, \mu_{i}\right)$ towards the steady state $\left(n_{+}, \mu_{+}\right)$holds true in the following sense:

$$
\left\{\begin{array}{l}
\left|\mu_{i}-\mu_{+}\right|=O\left(\frac{1}{\sqrt{1+t}}\right), \\
\left\|n_{i}(t, \cdot)-n_{+}\right\|_{L^{1}\left(\mathbb{R}_{+}\right)}=O\left(\frac{\sqrt{\log (1+t)}}{\sqrt{1+t}}\right),
\end{array}\right.
$$

where $n_{+}$is defined by (8). Moreover in the case of identical initial data $\left(n_{1}^{0}, \mu_{1}^{0}\right)=\left(n_{2}^{0}, \mu_{2}^{0}\right)=\left(n^{0}, \mu^{0}\right)$, we have a slightly better error estimate:

$$
\left\|n(t, \cdot)-n_{+}\right\|_{L^{1}\left(\mathbb{R}_{+}\right)}=O\left(\frac{1}{\sqrt{1+t}}\right) .
$$

On the other hand, for small initial conditions, or small parameter $\chi$, we prove the self-similar decay of the solution. To state this result, we introduce the notation $G$ for be the normalized Gaussian distribution on the half line

$$
G(x)=\sqrt{\frac{2}{\pi}} e^{-\frac{x^{2}}{2}}, \quad x \geq 0 .
$$

Theorem 1.3 (Self similar decay in the subcritical case). Let $\left(n_{i}, \mu_{i}\right)_{1 \leq i \leq 2}$ be the solution to (2)-(3)-(4) with initial data $\left(n_{i}^{0}, \mu_{i}^{0}\right)_{1 \leq i \leq 2}$ satisfying (5). Assume that

- either $\chi<4$,

- or that $\chi \geq 4$ and that there exists $\mu_{0} \in\left(0, \mu_{-}\right)$such that for $i=1,2$

$$
(\forall x \geq 0) \quad N_{i}^{0}(x) \leq N_{\mu_{0}}(x) .
$$

Assume in addition that

$$
\int_{0}^{\infty} n_{i}^{0}(x)\left(x^{2}+\log n_{i}^{0}(x)\right) \mathrm{d} x<+\infty .
$$

Then, for $i=1,2$, the following convergences hold true:

$$
\left\{\begin{array}{l}
\mu_{i}(t)=O\left(\frac{1}{\sqrt{1+t}}\right) \\
\left\|n_{i}(t, .)-\frac{1}{\sqrt{1+2 t}} G\left(\frac{\dot{\sqrt{1+2 t}}}{}\right)\right\|_{L^{1}\left(\mathbb{R}_{+}\right)}=O\left(\frac{1}{(1+t)^{1 / 4}}\right) .
\end{array}\right.
$$

We shall refer to the zero state as the "silent state" where no dialog takes place between the cells. On the other hand, we shall refer to the state $\left(n_{+}, \mu_{+}\right)$as the "dialog state", where the cells feel each other with a high fraction of activated protein at the cell boundary. Informally, our results state that, if $\chi>4$, then the system is bistable: if the cells do not invest enough in the communication with their partner, they do not respond to each other, and their molecular content spreads out; but a sufficient initial input from each cell in the dialog leads to the establishment of a stable activated state. The two stable states are separated by an unstable state associated with a low level of activation. Results are summarized in Table 1. Notice that not all the initial states are contained. In particular, we could not deal with the case where one cell has a high input whereas its partner has a low input initially: $N_{1}^{0}(x)<N_{\mu_{-}}<N_{2}^{0}(x)$. We expect that in this situation, both convergence may happen depending on the initial configuration.

The article is organized as follows: Section 2 is devoted to the biological assumptions underlying our model. A useful comparison principle is stated in Section 3. Section 4 is devoted to the proof of the non-linear stability 


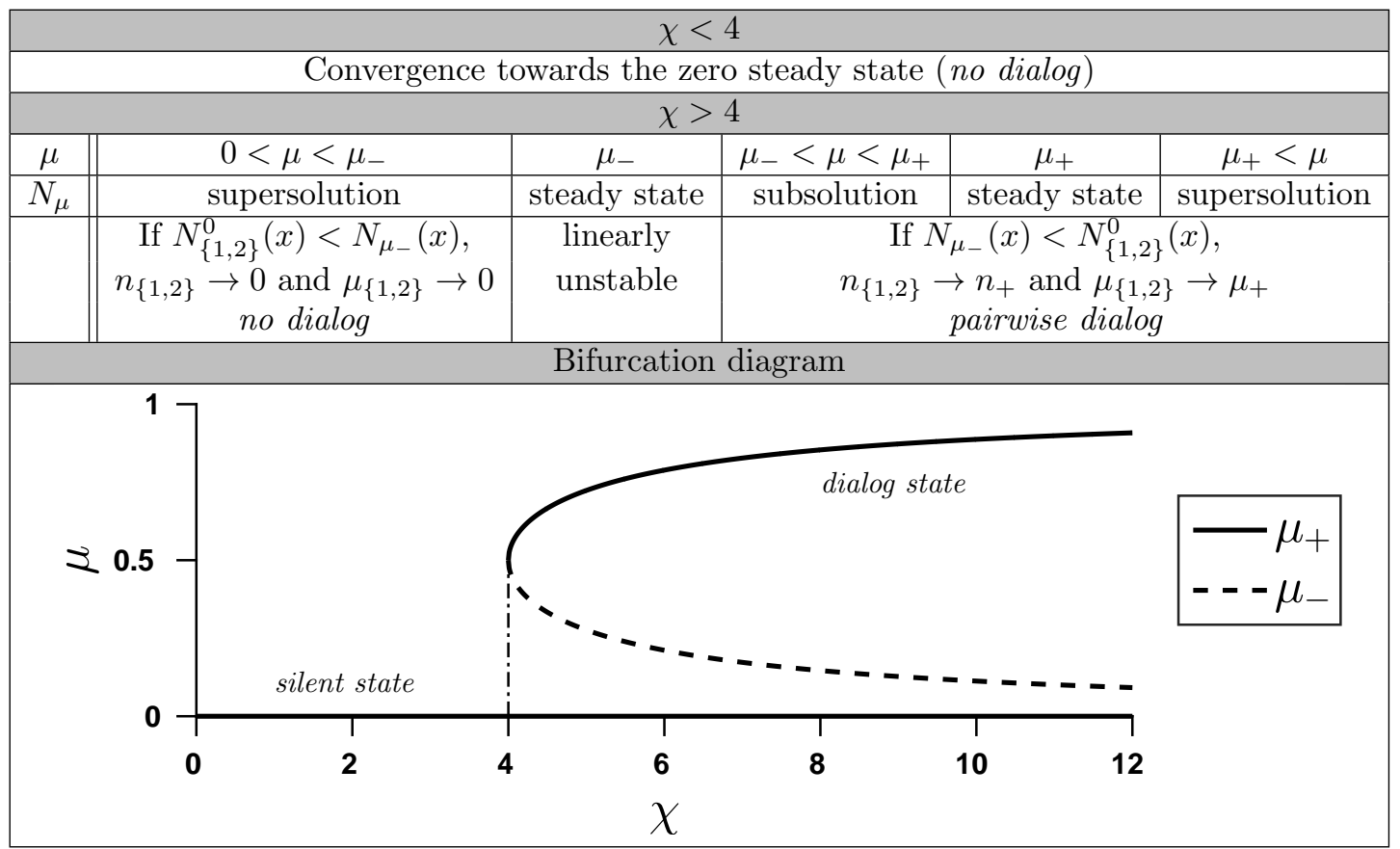

TABLE 1. Informal summary of the results and sketch of the bifurcation diagram. We refer to the main text for the exact statements and assumptions.

of the most concentrated steady state (Theorem 1.2). In Section 5, self-similar decay is establihed in the subcritical case (Theorem 1.3). Linear stability analysis is postponed to the Appendix (Proposition 1.1). Some notations and mathematical useful inequalities are recalled in the last section of the Appendix.

\section{Further BiologicAl BACKGROUnd}

Many events in plant and animal development depend on the ability of cells to interact with each other. Examples include the interaction of neuronal growth cones with target cells, cell division and differentiation, cell dissemination, inter-organ communications, tissue morphogenesis or regeneration.

Understanding how cells dynamically integrate and respond to external signals from the microenvironment is a very challenging question. From the biological viewpoint, a generic model for cell communication is given by yeast cell mating. Yeast cell communication involves some intra-cellular proteins (as Cdc42), the cell cytoskeleton and extra-cellular pheromone molecules, Fig. 2 1.

Several mathematical models have been proposed in the past decade. They incorporate many aspects of the molecular mechanisms involved in pheromone-induced protein aggreagtion. Although some of these models have been tested for their ability to fit quantitative data [12,14, 17, 19, 23, 26, 30, 31, 33, they have not been quantitatively assessed for their ability to make accurate predictions with no additional free parameter. In the model presented here, we focus on the intracellular actin-mediated transport of the protein Cdc42 which was shown to contribute to the initiation of cell polarization via inhomogeneous protein distribution at the cell surface [38, 39, but see 27, 33, 36] for a recent discussion about this hypothesis. Other factors, such as the Bem1-mediated positive feedback e.g., are not present in the model considered here. Indeed, we believe it is of interest to investigate the potentiality of a mass-preserving mechanism to account for symmetry breaking in the cell content. 

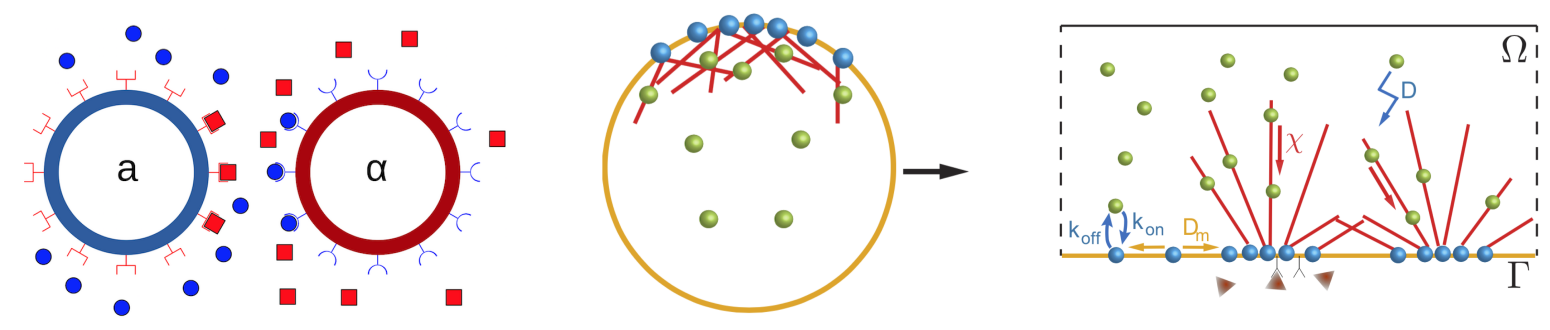

FiguRE 2. (Left) Yeast cells of both types (a or $\alpha)$ secrete some pheromone $\left(S_{\mathbf{a}}\right.$ or $\left.S_{\alpha}\right)$ and bear a pheromone receptor to detect the signal from the opposite cell type. (Right) A twodimensional cartoon of protein dynamics inside a cell. Actin is polymerized into short filaments, that interact with each other. They are bundled together to form actin cables (which are part of the cytoskeleton) that cross the cell. In our model, the nucleation of filaments is influenced both by the concentration of Cdc42 at the membrane (the proteins that are actively transported along actin filaments) and by the concentration of external pheromone.

The present work is the continuation of our analysis program about a model which was first introduced in [21], then studied in 8, 9] and finally tested for its ability to predict experimental data in [32] at the level of a single cell. This model relies on a coarse-grained description of actin-mediated transport and it is expressed by a non-linear and non-local partial differential equation. Here, we enrich the single cell model, and we push further our mathematical analysis in order to study pairwise cell-cell communication.

Although the present study is restricted to the one-dimensional setting, it is instructive to describe a higher dimensional situation, say the two-dimensional setting for the sake of simplicity. Denoting respectively by $n(t, x)$ the concentration of the Cdc42 protein and by $c(t, x)$ the density of actin filaments in the cytoplasm of the cell (the bounded domain $\Omega \subset \mathbb{R}^{2}$ ), and denoting by $\mu(t, s)$ the concentration of Cdc42 on the membrane of the cell $(\Gamma=\partial \Omega)$, the model is:

$$
\begin{cases}\partial_{t} n=\Delta n-\chi \nabla \cdot(n \nabla c), & \text { in } \Omega, \\ \partial_{t} \mu=\partial_{s s} \mu+n-\mu, & \text { on } \Gamma,\end{cases}
$$

where $s$ is a parametrisation of the boundary $\Gamma$.

The advection term $\chi \nabla c$ accounts for active transport of proteins along actin filaments. Nucleation of new filaments is assumed to occur at the plasma membrane, under the combined action of Cdc42 and external pheromone molecules. After a dimensional analysis, the model that describes the actin filaments density is the following one:

$$
\begin{cases}-\Delta c=0, & \text { in } \Omega, \\ -\nabla c \cdot \mathbf{e}=S \mu, & \text { on } \Gamma,\end{cases}
$$

where $\mathbf{e}$ is the unit outward normal vector and $S(t, s)$ is the trace of the pheromone external signal on the cell membrane. Equations $\sqrt{11}$ and $\sqrt{12}$ are complemented by initial conditions and by an additional boundary condition on the cell membrane which guarantees the conservation of the total Cdc42 pool in the cell:

$$
(\nabla n-\chi n \nabla c) \cdot \mathbf{e}=-\partial_{t} \mu \quad \text { on } \Gamma
$$

In the one dimensional case where the cytoplasm of the cell is modelled by the half line $\Omega=\mathbb{R}_{+}$, and the membrane is located at $x=0$, the model $(11)-(12)$ simply read as

$$
\partial_{t} n=\partial_{x x} n+S \mu \partial_{x} n, \quad t>0, \quad x>0,
$$


with an additionnal flux boundary condition that assures mass conservation. This latter equation has been analyzed in [8, 9, 28, its dynamics is well understood and is reminiscent of the Keller-Segel model in two dimensions. The principal result of [8] was to identify regimes in which non homogeneous stationary states, that were interpreted as polarised states, emerge.

In nature, the budding yeast, Saccharomyces cerevisiae, exists either in diploid state, or in haploid state with two possible types (a or $\alpha$ ). Cells of both types secrete some pheromone $\left(S_{\mathbf{a}}\right.$ or $\left.S_{\alpha}\right)$, Fig. 2 , and bear a pheromone receptor to detect the pheromone produced by the cells of the opposite type, 22]. In the present work we propose a model where the release of extra-cellular pheromone $\left(S_{\mathbf{a}}\right.$ or $\left.S_{\alpha}\right)$ depends on the concentration of the protein Cdc42 at the membrane [34]. Furthermore, according to the biological litterature, 3.29, 35 36], we assume that the pheromone contributes to the nucleation of new filaments at the plasma membrane of the cell of the opposite type, see Fig 2. To describe the protein dynamics on each cell membrane we use and enrich the model (13) in the one-dimensional case. This leads to a coupled system of two non-linear transport-diffusion equations set in two opposite half-lines. Throughout this work, the cell type is referred to by the subscript $i=1,2$.

This additional level of cellular pairwise communication leads to the following rectification of the model (13): for the cell $i$, we consider that the advection field is $-\chi S_{j}(t) \mu_{i}(t)$ where $S_{j}$ is the concentration of pheromone produced by the cell of the opposite type $j$. Moreover, in this work we postulate that $S_{j}$ is proportional to $\mu_{j}$, which means that the pheromone produced by cell of type $i$ is influenced by the level of activation of the opposite cell, possibly discounted by a damping factor depending on the inter-cellular distance (not explicitly written here). Thus, in both cells the advection field is the same: $-\chi \mu_{1}(t) \mu_{2}(t)$. From the biological point of view this model describes whether proteins Cdc42 will mainly get attached to the membrane, or will mainly diffusive in the bulk. The former event will be referred to as a "polarized state" for each cell, as in [8, 9, 32. The case where both cells are polarized will be referred to as the "dialog state" between the two cells.

\section{THE COMPARISON PRINCIPLE AND ITS CONSEQUENCES}

We start noticing that there is no direct comparison principle on 22-(3)-4 4 . In this section, we first establish a concentration comparison principle on the cumulated distribution functions $N_{i}$ (6), reminiscent of [28], and analogous to the radially symmetric Keller-Segel system, see for instance [4, 24 and references therein. In a second step, we identify a family of sub/supersolutions that play a pivotal role in the non-linear stability analysis.

\subsection{The concentration-comparison principle}

For $i=1,2$, the integrated quantities $N_{i}(t, x)=\mu_{i}(t)+\int_{0}^{x} n_{i}(t, y) \mathrm{d} y$ associated with (2)-(3)-(4) and (5) satisfy

$$
\left\{\begin{array}{l}
\partial_{t} N_{i}(t, x)-\partial_{x x} N_{i}(t, x)-\chi \mu_{1}(t) \mu_{2}(t) \partial_{x} N_{i}(t, x)=0 \\
N_{i}(t, 0)=\mu_{i}(t), \quad \lim _{x \rightarrow \infty} N_{i}(t, x)=1 \\
\frac{\mathrm{d} \mu_{i}}{\mathrm{~d} t}(t)=\partial_{x} N_{i}(t, 0)-\mu_{i}(t)
\end{array}\right.
$$

Definition 3.1. A supersolution (resp. subsolution) to (14) is a couple of nondecreasing functions (with respect to $x)\left(\bar{N}_{1}, \bar{N}_{2}\right)$ (resp. $\left.\left(\underline{N}_{1}, \underline{N}_{2}\right)\right)$ satisfying

$$
\left\{\begin{array}{l}
\partial_{t} \bar{N}_{i}(t, x)-\partial_{x x} \bar{N}_{i}(t, x)-\chi \bar{\mu}_{1}(t) \bar{\mu}_{2}(t) \partial_{x} \bar{N}_{i}(t, x) \geq 0 \\
\bar{N}_{i}(t, 0)=\bar{\mu}_{i}(t), \quad \lim _{x \rightarrow \infty} \bar{N}_{i}(t, x)=1 \\
\frac{\mathrm{d} \bar{\mu}_{i}}{\mathrm{~d} t}(t) \geq \partial_{x} \bar{N}_{i}(t, 0)-\bar{\mu}_{i}(t)
\end{array}\right.
$$

with similar definition for a subsolution by changing $\geq$ into $\leq$.

We now state the concentration comparison principle. 
Lemma 3.2 (Comparison principle). Let $\left(\bar{N}_{1}, \bar{N}_{2}\right)$ and $\left(\underline{N}_{1}, \underline{N}_{2}\right)$ be respectively smooth super and subsolution to (14) defined on $[0, T] \times \mathbb{R}_{+}$. Assume that for $i=1,2$

$$
(\forall x \geq 0) \quad \underline{N_{i}}(0, x) \leq \bar{N}_{i}(0, x), \quad \text { and } \quad \bar{\mu}_{i}(0)>\underline{\mu}_{i}(0) .
$$

Then, the following inequalities hold true for $i=1,2$,

$$
(\forall t \in(0, T))(\forall x \geq 0) \quad \underline{N_{i}}(t, x) \leq \bar{N}_{i}(t, x) .
$$

Proof. For $i=1,2$, denoting $F_{i}=\bar{N}_{i}-\underline{N}_{i}$, one has

$$
\left\{\begin{array}{l}
\partial_{t} F_{i}-\partial_{x x} F_{i}-\chi \bar{\mu}_{1}(t) \bar{\mu}_{2}(t) \partial_{x} F_{i} \geq \chi\left(\bar{\mu}_{1}(t) \bar{\mu}_{2}(t)-\underline{\mu}_{1}(t) \underline{\mu}_{2}(t)\right) \partial_{x} \underline{N}_{i}, \\
F_{i}(t, 0)=\bar{\mu}_{i}(t)-\underline{\mu}_{i}(t), \quad \lim _{x \rightarrow \infty} F_{i}(t, x)=0 \\
\frac{\mathrm{d}}{\mathrm{d} t}\left(\bar{\mu}_{i}(t)-\underline{\mu}_{i}(t)\right) \geq \partial_{x} F_{i}(t, 0)-\left(\bar{\mu}_{i}(t)-\underline{\mu}_{i}(t)\right) \\
F_{i}(0, x)=\bar{N}_{i}(0, x)-\underline{N_{i}}(0, x) \geq 0
\end{array}\right.
$$

The bootstrap acts as follows. Since we are dealing with nondecreasing functions $N_{i}$, the quantity $\chi\left(\bar{\mu}_{1}(t) \bar{\mu}_{2}(t)-\underline{\mu}_{1}(t) \underline{\mu}_{2}(t)\right) \partial_{x} \underline{N}_{i}$ is nonnegative as long as

$$
\bar{\mu}_{1}(t) \bar{\mu}_{2}(t)-\underline{\mu}_{1}(t) \underline{\mu}_{2}(t) \geq 0
$$

holds true. This is in particular the case if

$$
\bar{\mu}_{1}(t) \geq \underline{\mu}_{1}(t) \quad \text { and } \quad \bar{\mu}_{2}(t) \geq \underline{\mu}_{2}(t) .
$$

Recalling the assumption $\bar{\mu}_{i}(0)>\underline{\mu}_{i}(0)$, we denote by $\tau>0$ the first time for which an equality in 15 occurs. Suppose without loss of generality that $\bar{\mu}_{1}(\tau)=\underline{\mu}_{1}(\tau)$. Let us define the function $z(t)$ by

$$
z(t):=\bar{\mu}_{1}(t)-\underline{\mu}_{1}(t) .
$$

Then, for all $(t, x) \in(0, \tau) \times(0, \infty)$, one has

$$
\left\{\begin{array}{l}
\partial_{t} F_{1}(t, x)-\partial_{x x} F_{1}(t, x)-\chi \bar{\mu}_{1}(t) \bar{\mu}_{2}(t) \partial_{x} F_{1}(t, x) \geq 0 \\
F_{1}(t, 0)=\bar{\mu}_{1}(t)-\underline{\mu}_{1}(t)=z(t) \geq 0, \quad \lim _{x \rightarrow \infty} F_{1}(t, x)=0 \\
\frac{\mathrm{d} z}{\mathrm{~d} t}(t) \geq \partial_{x} F_{1}(t, 0)-z(t)
\end{array}\right.
$$

Recalling in addition the assumption $F_{i}(0,0)>0$, it follows that there exists a nonnegative compactly supported (in $(0,+\infty)$ ) function $f \neq 0$ such that $f(x) \leq F_{1}(0, x)$ and $f(0)=0$. Next, we consider the solution to the parabolic equation

$$
\left\{\begin{array}{l}
\partial_{t} g(t, x)-\partial_{x x} g(t, x)-\chi \bar{\mu}_{1}(t) \bar{\mu}_{2}(t) \partial_{x} g(t, x)=0, \\
g(t, 0)=0 \\
g(0, x)=f(x)
\end{array}\right.
$$

On the one hand, it follows from the standard maximum principle 18 that $F_{1} \geq g$ on $[0, \tau] \times[0, \infty)$. On the other hand, classical parabolic regularity implies that $g(t, x)>0$ for all $(t, x) \in(0, \tau] \times(0, \infty)$. Hence, applying the Hopf Lemma [18, it follows that $\partial_{x} g(\tau, 0)>0$. Next, from the equality $F_{1}(\tau, 0)=g(\tau, 0)=0$, we deduce that $\partial_{x} F_{1}(\tau, 0) \geq \widehat{\partial_{x}} g(\tau, 0)>0$. Consequently one has $\left.\frac{\mathrm{d}}{\mathrm{d} t} z(t)\right|_{t=\tau}>0$ which contradicts $z>0$ on $[0, \tau)$ and $z(\tau)=0$. 


\subsection{Remarkable sub/supersolutions}

For any parameter $\mu \in(0,1)$, the pair $\left(N_{\mu}, N_{\mu}\right)$ is either a subsolution or a supersolution, depending on the sign of $P(\mu)$ with $P$ defined by $(7)$ and $N_{\mu}(x)=\mu+(1-\mu)\left(1-e^{-\chi \mu^{2} x}\right)$.

Lemma 3.3. The pair $\left(N_{\mu}, N_{\mu}\right)$ where

$$
N_{\mu}(x)=\mu+(1-\mu)\left(1-e^{-\chi \mu^{2} x}\right),
$$

is a supersolution (resp. a subsolution) to (14) if

$$
P(\mu)=\chi \mu^{2}-\chi \mu+1 \geq 0 \quad(\text { resp. } \quad \leq 0) .
$$

Recall that $P(\mu) \leq 0$, hence $\left(N_{\mu}, N_{\mu}\right)$ is a subsolution, if and only if $\mu \in\left[\mu_{-}, \mu_{+}\right]$. However the interval is empty if $\chi<4$. Indeed, in the latter case, the pair $\left(N_{\mu}, N_{\mu}\right)$ is always a supersolution. The various cases are reported in Table 1.

Proof. A straightforward computation yields the following identities:

$$
\left\{\begin{array}{l}
\partial_{t} N_{\mu}-\partial_{x x} N_{\mu}-\chi \mu^{2} \partial_{x} N_{\mu}=0 \\
N_{\mu}(0)=\mu \\
\partial_{x} N_{\mu}(0)-\mu=(1-\mu) \chi \mu^{2}-\mu=-\mu\left(\chi \mu^{2}-\chi \mu+1\right) .
\end{array}\right.
$$

The conclusion follows from the definition of $P(7)$.

\section{Non-linear stability of $\left(n_{+}, \mu_{+}\right)$: Proof of Theorem 1.2}

We split the proof in several steps. We begin with the symmetric case where the cells are identical: $\left(n_{1}, \mu_{1}\right)=$ $\left(n_{2}, \mu_{2}\right)$. Then, the result is extended to the general case by using the comparison principle.

\subsection{The symmetric case}

In this part, we assume that $\left(n_{1}^{0}, \mu_{1}^{0}\right)=\left(n_{2}^{0}, \mu_{2}^{0}\right)=\left(n^{0}, \mu^{0}\right)$, so that the solutions $\left(n_{1}, \mu_{1}\right)$ and $\left(n_{2}, \mu_{2}\right)=(n, \mu)$ are always the same. We keep all the assumptions, in particular (5), i.e. $M_{i}=1$. We drop the subscript $i=1,2$ for the sake of clarity. Our goal is to prove Theorem 1.2 in the symmetric case.

$$
\left\{\begin{array}{l}
\partial_{t} n(t, x)-\partial_{x x} n(t, x)-\chi \mu(t)^{2} \partial_{x} n(t, x)=0, \quad(t, x) \in(0, \infty)^{2}, \\
\partial_{x} n(t, 0)+\chi \mu(t)^{2} n(t, 0)=\frac{\mathrm{d} \mu}{\mathrm{d} t}(t)=n(t, 0)-\mu(t)
\end{array}\right.
$$

The main argument is based on the construction of a suitable functional that decays along the flow under some conditions. It is a mixture of entropy and quadratic scalar contributions, similarly as for the case of a single cell [28], see Lemma 4.1 below. Next, the assumptions made on the initial condition allow to obtain lower bounds on the terms involved in entropy dissipation and to prove that the Lyapunov functional tends to zero. The keystone is to establish that $\mu$ stays away from $\mu_{-}$in order to control the relaxation of $\mu(t)$ towards $\mu_{+}$.

We define the functional $L$ by:

$$
L(t):=(1-\mu(t)) \mathcal{H}\left(m(t, \cdot) \mid m_{+}\right)=\int_{0}^{\infty} n(t, x) \log \frac{m(t, x)}{m_{+}(x)} \mathrm{d} x \geq 0,
$$

where $m_{+}$is given by $[8], \mathcal{H}(n \mid p)$ is the relative entropy [2], and

$$
m(t, x)=\frac{n(t, x)}{\int_{0}^{\infty} n(t, x) \mathrm{d} x}=\frac{n(t, x)}{1-\mu(t)},
$$


is the renormalized density in the bulk. In addition, the function $f$ is defined by

$$
f(\mu)=\mu \log \frac{\mu}{\mu_{+}}+(1-\mu) \log \frac{1-\mu}{1-\mu_{+}}+\chi \frac{\left(\mu-\mu_{+}\right)^{2}\left(\mu+2 \mu_{+}\right)}{3} .
$$

The function $f$ is designed such as $f\left(\mu_{+}\right)=0$, and

$$
f^{\prime}(\mu)=\log \frac{\mu\left(1-\mu_{+}\right)}{\mu_{+}(1-\mu)}+\chi\left(\mu^{2}-\mu_{+}^{2}\right)
$$

In particular, the function $f$ is nonincreasing on $\left(0, \mu_{+}\right)$and nondecreasing on $\left(\mu_{+}, 1\right)$, hence it is nonnegative on $(0,1)$.

Lemma 4.1. Let $(n, \mu)$ be the solution to (16). The following inequality holds

$$
\frac{\mathrm{d}}{\mathrm{d} t}(L(t)+f(\mu(t))) \leq-D(t)^{2}-\chi\left(\mu(t)^{2}-\mu_{+}^{2}\right) \mu(t) P(\mu(t))
$$

where $D^{2}$ is defined as

$$
D^{2}=\int_{0}^{\infty} n\left(\partial_{x} \log n+\chi \mu^{2}\right)^{2} \mathrm{~d} x=(1-\mu) \mathcal{I}\left(m \mid \chi \mu^{2} e^{-\chi \mu^{2} x}\right)
$$

and $\mathcal{I}(p \mid q)=\int_{0}^{\infty} p\left(\partial_{x} \log \frac{p}{q}\right)^{2} \mathrm{~d} x$ is the Fisher information.

Proof of Lemma 4.1. The functional $L$ can be rearranged as follows,

$$
\begin{aligned}
L & =\int_{0}^{\infty}\left(n \log n-n \log m_{+}-n \log (1-\mu)\right) \mathrm{d} x \\
& =\int_{0}^{\infty}\left(n \log n-n \log m_{+}\right) \mathrm{d} x-(1-\mu) \log (1-\mu) .
\end{aligned}
$$

Hence, differentiating $L$, we obtain

$$
\frac{\mathrm{d} L}{\mathrm{~d} t}=\int_{0}^{\infty} \partial_{t} n\left(1+\log n-\log m_{+}\right) \mathrm{d} x+(1+\log (1-\mu)) \frac{\mathrm{d} \mu}{\mathrm{d} t},
$$

and using that $\int_{0}^{\infty} n \mathrm{~d} x+\mu=1$, we get

$$
\frac{\mathrm{d} L}{\mathrm{~d} t}=\int_{0}^{\infty} \partial_{t} n \log \frac{n}{m_{+}} \mathrm{d} x+\log (1-\mu) \frac{\mathrm{d} \mu}{\mathrm{d} t}
$$


Next, using the definition of $m_{+}(8)$, that $(n, \mu)$ is solution to $(16)$, an integration by parts yields the following identities:

$$
\begin{aligned}
\frac{\mathrm{d} L}{\mathrm{~d} t} & =\int_{0}^{\infty} \log \frac{n}{m_{+}}\left(\partial_{x x} n+\chi \mu^{2} \partial_{x} n\right) \mathrm{d} x+\log (1-\mu) \frac{\mathrm{d} \mu}{\mathrm{d} t} \\
& =-\left(\log \frac{n(t, 0)}{m_{+}(0)}\right) \frac{\mathrm{d} \mu}{\mathrm{d} t}-\int_{0}^{\infty} n\left(\partial_{x} \log n+\chi \mu_{+}^{2}\right)\left(\partial_{x} \log n+\chi \mu^{2}\right) \mathrm{d} x+\log (1-\mu) \frac{\mathrm{d} \mu}{\mathrm{d} t} \\
& =-\left(\log \frac{n(t, 0)}{(1-\mu) m_{+}(0)}\right) \frac{\mathrm{d}}{\mathrm{d} t} \mu-\underbrace{\int_{0}^{\infty} n\left(\partial_{x} \log n+\chi \mu^{2}\right)^{2} \mathrm{~d} x}_{=D^{2}}+\chi\left(\mu^{2}-\mu_{+}^{2}\right) \int_{0}^{\infty}\left(\partial_{x} n+\chi \mu^{2} n\right) \mathrm{d} x \\
& =-D^{2}-\left(\log \frac{n(t, 0)}{(1-\mu) m_{+}(0)}\right) \frac{\mathrm{d} \mu}{\mathrm{d} t}+\chi\left(\mu^{2}-\mu_{+}^{2}\right)\left(-n(t, 0)+\chi \mu^{2}(1-\mu)\right) .
\end{aligned}
$$

Using now that $\frac{\mathrm{d}}{\mathrm{d} t} \mu=n(t, 0)-\mu$, we deduce that

$$
\begin{aligned}
\frac{\mathrm{d} L}{\mathrm{~d} t}= & -D^{2}+\underbrace{\left(\log \frac{\mu}{n(t, 0)}+\log \frac{(1-\mu) m_{+}(0)}{\mu}\right) \frac{\mathrm{d} \mu}{\mathrm{d} t}+\chi\left(\mu^{2}-\mu_{+}^{2}\right)\left(-\frac{\mathrm{d} \mu}{\mathrm{d} t}-\mu+\chi \mu^{2}(1-\mu)\right)}_{\leq 0} \\
= & -D^{2}+\underbrace{(n(t, 0)-\mu) \log \frac{\mu}{n(t, 0)}}+\chi\left(\mu^{2}-\mu_{+}^{2}\right) \mu\left(-\chi \mu^{2}+\chi \mu-1\right) \\
& +\left(\chi \mu_{+}^{2}-\chi \mu^{2}+\log \frac{(1-\mu) \chi \mu_{+}^{2}}{\mu}\right) \frac{\mathrm{d} \mu}{\mathrm{d} t} .
\end{aligned}
$$

The conclusion follows by using the definitions of $f(17)$ and $P(7)$, which in turn implies $\chi \mu_{+}^{2}=\frac{\mu_{+}}{1-\mu_{+}}$.

An important observation is that, for all $\mu \in\left[\mu_{-}, 1\right]$, the quantity $\left(\mu^{2}-\mu_{+}^{2}\right) P(\mu)$ is nonnegative. As a consequence, the second contribution in the r.h.s. of (18) is dissipative provided $\mu(t)$ remains above $\mu_{-}$. Such an a priori control enables to prove that $L+f$ converges to zero with an algebraic rate.

Lemma 4.2. Under the same assumptions as in Theorem 1.2, there exists a constant $C$ depending on $\left(\underline{\mu}_{0}, \bar{\mu}_{0}\right)$ such that

$$
\frac{\mathrm{d}}{\mathrm{d} t}\left(L(t)+f(\mu(t)) \leq-C\left(L(t)+f(\mu(t))^{2} .\right.\right.
$$

Proof of Lemma 4.2. We first note that $N_{\underline{\mu}_{0}}(x)$ is a subsolution, whereas $N_{\bar{\mu}_{0}}(x)$ is a supersolution, see Lemma 3.3 and (9) (see also Table 1). As the initial data are well ordered by assumption (10), we deduce from the comparison principle in Lemma 3.2 that

$$
\forall(t, x) \in(0, \infty) \times \mathbb{R}_{+}, \quad N_{\underline{\mu}_{0}}(x) \leq N(t, x) \leq N_{\bar{\mu}_{0}}(x) .
$$

In particular, we have $\mu(t) \geq \underline{\mu}_{0}>\mu_{-}$. Hence, $\left(\mu(t)^{2}-\mu_{+}^{2}\right) P(\mu(t))$ is nonnegative. Furthermore, the family of functions $N_{\mu}$ is increasing with respect to $\mu$, thus

$$
N_{\underline{\mu}_{0}}(x) \leq \mu(t)+(1-\mu(t))\left(1-e^{-\chi \mu(t)^{2} x}\right) \leq N_{\bar{\mu}_{0}}(x) .
$$

Using the quadratic Wasserstein distance $W$, whose definition is recalled in the Appendix, together with the definition of $m(t, x)=\frac{n(t, x)}{\int_{0}^{\infty} n(t, x) \mathrm{d} x}=\frac{n(t, x)}{1-\mu(t)}$, we see that

$$
W\left(m, \chi \mu^{2} e^{-\chi \mu^{2} x}\right)=\frac{1}{\sqrt{1-\mu}} W\left(\mu \delta_{0}+(1-\mu) m, \mu \delta_{0}+(1-\mu) \chi \mu^{2} e^{-\chi \mu^{2} x}\right),
$$


where $\delta_{0}$ is the Dirac mass in $x=0$. The bounds on $N$ and $N_{\mu}$ in 190 and $(20)$, allow to apply lemma $\mathrm{B} .3$ to obtain

where

$$
W\left(\mu \delta_{0}+(1-\mu) m, \mu \delta_{0}+(1-\mu) \chi \mu^{2} e^{-\chi \mu^{2} x}\right) \leq C_{W}\left(\underline{\mu}_{0}, \bar{\mu}_{0}\right),
$$

$$
C_{W}\left(\underline{\mu}_{0}, \bar{\mu}_{0}\right)=W\left(\underline{\mu}_{0} \delta_{0}+\left(1-\underline{\mu}_{0}\right) \chi \underline{\mu}_{0}^{2} e^{-\chi \underline{\mu}_{0}^{2} x}, \bar{\mu}_{0} \delta_{0}+\left(1-\bar{\mu}_{0}\right) \chi \bar{\mu}_{0}^{2} e^{-\chi \bar{\mu}_{0}^{2} x}\right)<+\infty .
$$

Then, applying the HWI inequality, see Lemma B.6 in the Appendix, to the exponential measure $\chi \mu^{2} e^{-\chi \mu^{2} x}$, we obtain that

$$
\begin{aligned}
D^{2} & =(1-\mu) \mathcal{I}\left(m \mid \chi \mu^{2} e^{-\chi \mu^{2} x}\right) \geq(1-\mu)\left(\frac{\mathcal{H}\left(m \mid \chi \mu^{2} e^{-\chi \mu^{2} x}\right)}{W\left(m, \chi \mu^{2} e^{-\chi \mu^{2} x}\right)}\right)^{2} \\
& \geq(1-\mu)^{2}\left(\frac{\mathcal{H}\left(m \mid \chi \mu^{2} e^{-\chi \mu^{2} x}\right)}{C_{W}\left(\underline{\mu}_{0}, \bar{\mu}_{0}\right)}\right)^{2} .
\end{aligned}
$$

Recalling the definition of the relative entropy, $\mathcal{H}(n \mid p)=\int_{0}^{\infty} n \log (n / p) \mathrm{d} x$, we see that

$$
\mathcal{H}\left(m \mid \chi \mu^{2} e^{-\chi \mu^{2} x}\right)=\frac{1}{1-\mu}(\underbrace{(1-\mu) \mathcal{H}\left(m \mid m_{+}\right)}_{=L}+\Delta(\mu))
$$

where the correction $\Delta(\mu)$ is defined by

$$
\Delta(\mu)=(1-\mu)\left(\log \frac{\mu_{+}^{2}}{\mu^{2}}\right) \int_{0}^{\infty} m \mathrm{~d} x+\chi(1-\mu)\left(\mu^{2}-\mu_{+}^{2}\right) \int_{0}^{\infty} x m \mathrm{~d} x .
$$

We deduce from (18) and (21) that

$$
\frac{\mathrm{d}}{\mathrm{d} t}(L+f(\mu)) \leq-\frac{(L+\Delta(\mu))^{2}}{(1-\mu) C_{W}\left(\underline{\mu}_{0}, \bar{\mu}_{0}\right)^{2}}-\chi\left(\mu^{2}-\mu_{+}^{2}\right) \mu P(\mu) \leq 0 .
$$

Noticing additionally that

$$
(L+f)^{2}=(L+\Delta)^{2}+(f-\Delta)^{2}+2(L+\Delta)(f-\Delta) \leq\left(1+\frac{1}{\alpha}\right)(L+\Delta)^{2}+(1+\alpha)(f-\Delta)^{2},
$$

we see that for any $\alpha>0$, we have

$$
\frac{\mathrm{d}}{\mathrm{d} t}(L+f(\mu)) \leq \frac{-1}{(1-\mu) C_{W}\left(\underline{\mu}_{0}, \bar{\mu}_{0}\right)^{2}}\left(\frac{\alpha}{1+\alpha}(L+f(\mu))^{2}-\alpha(f(\mu)-\Delta(\mu))^{2}+R(\mu)\right),
$$

where $R(\mu)=\chi C_{W}\left(\underline{\mu}_{0}, \bar{\mu}_{0}\right)^{2} \mu(1-\mu)\left(\mu^{2}-\mu_{+}^{2}\right) P(\mu)$.

It is useful to notice that $\int x m \mathrm{~d} x$ is uniformly bounded, as it follows from the estimate 19$)$ together with an integration by parts:

$$
\int_{0}^{\infty} x m(t, x) \mathrm{d} x=\frac{1}{1-\mu} \int_{0}^{\infty}(1-N(t, x)) \mathrm{d} x \leq \frac{1}{1-\bar{\mu}_{0}} \int_{0}^{\infty}\left(1-N_{\underline{\mu}_{0}}\right) \mathrm{d} x=\frac{1-\underline{\mu}_{0}}{\chi \underline{\mu}_{0}^{2}\left(1-\bar{\mu}_{0}\right)} .
$$


Therefore, both $|\Delta|$ and $|f|$ are $O\left(\left|\mu-\mu_{+}\right|\right)$in $\left(\underline{\mu}_{0}, 1\right)$. we deduce that $(f(\mu)-\Delta(\mu))^{2}=O(R(\mu))$ in a neighbourhood of $\mu_{+}$, as $\mu_{+}$is a root of $P$. Hence, there exists a constant $\alpha_{0}>0$ such that

$$
\left(\forall \mu \in\left(\underline{\mu}_{0}, 1\right)\right) \quad-\alpha_{0}(f(\mu)-\Delta(\mu))^{2}+R(\mu) \geq 0
$$

Choosing $C=\frac{\alpha_{0}}{\left(1+\alpha_{0}\right)\left(1-\underline{\mu}_{0}\right) C_{W}\left(\underline{\mu}_{0}, \bar{\mu}_{0}\right)^{2}}$, this achieves the proof of Lemma 4.2

Corollary 4.3. Under the same assumptions as in Theorem 1.2, the following estimates hold

$$
(\forall t>0), \quad 0 \leq \int_{0}^{\infty} n(t, x) \log \frac{m(t, x)}{m_{+}(x)} \mathrm{d} x+\left(\mu(t)-\mu_{+}\right)^{2} \leq \frac{C}{1+t},
$$

and

$$
\forall t>0, \quad\left\|n-n_{+}\right\|_{L^{1}\left(\mathbb{R}_{+}\right)} \leq \frac{C}{\sqrt{1+t}},
$$

where $n_{+}=\left(1-\mu_{+}\right) m_{+}(8)$.

Proof. The first inequality is a direct consequence of lemma 4.2 . The second one is obtained by the triangle inequality:

$$
\left\|n-\left(1-\mu_{+}\right) m_{+}\right\|_{L^{1}\left(\mathbb{R}_{+}\right)} \leq(1-\mu)\left\|m-m_{+}\right\|_{L^{1}\left(\mathbb{R}_{+}\right)}+\left|\mu-\mu_{+}\right|,
$$

We apply finally the Csiszar-Kullbáck inequality (see proposition B.7):

$$
\left\|m-m_{+}\right\|_{L^{1}\left(\mathbb{R}_{+}\right)} \leq \sqrt{2 \mathcal{H}\left(m \mid m_{+}\right)} \leq \sqrt{\frac{2 L(t)}{1-\mu(t)}}
$$

from which we can deduce 24, based on $(23)$.

\subsection{Refined estimates of convergence for special solutions}

On the way to extending the previous result to the general, non symmetric case, further estimates of convergence are required. Below, we focus on two families of solutions, those which are initialized with $N_{\underline{\mu}_{0}}(x)$, resp. $N_{\bar{\mu}_{0}}(x)$, where $\underline{\mu}_{0}$ and $\bar{\mu}_{0}$ are as in Theorem 1.2 . This is of particular interest as any solution of the general problem with suitable initial data fits in between these two solutions, due to the comparison principle 3.2 . For the sake of brevity, we denote by $(\mu, \underline{n})$, resp. $(\bar{\mu}, \bar{n})$ the solution of the symmetric problem with the initial data corresponding to $N_{\underline{\mu}_{0}}(x)$, resp. $N_{\bar{\mu}_{0}}(x)$.

Corollary 4.3 asserts that the error $\mu(t)-\mu_{+}$is of order $(1+t)^{-1 / 2}$ at most. However, it is possible to gain a better rate of convergence for $(\bar{\mu}, \bar{n})$, in integral form, or alternatively in the Wasserstein distance $W_{1}$ (which accounts for a weaker convergence than the $L^{1}$ norm).

Lemma 4.4. Under the same assumptions as in Theorem 1.2, the error $\left(\bar{\mu}-\mu_{+}\right)$belongs to $L^{1}\left(\mathbb{R}_{+}\right)$. Furthermore, there exist a constant $C$, such that the following estimates hold true

$$
\begin{aligned}
\int_{t}^{\infty}\left|\bar{\mu}(s)-\mu_{+}\right| \mathrm{d} s & \leq \frac{C}{\sqrt{1+t}}, \\
W_{1}\left(\bar{\mu} \delta_{0}+\bar{n}, \mu_{+} \delta_{0}+n_{+}\right) & \leq \frac{C}{\sqrt{1+t}} . \\
\left|\bar{\mu}-\mu_{+}\right| & \leq \frac{C}{\sqrt{1+t}} .
\end{aligned}
$$

Similar results hold for $(\underline{\mu}, \underline{n})$. 
Proof. The last inequality (27) follows from (23) which was established in corollary 4.3. Let us start with the first point of the Lemma. Since $\bar{\mu}-\mu_{+} \geq 0$, the absolute value can be removed in (25). Recall that the Wasserstein distance $W_{1}$ involves the $L^{1}$ norm of the cumulated distribution function: if we define $\bar{N}$ and $N_{+}$ as in (6) for $(\bar{\mu}, \bar{n})$ and $\left(\mu_{+}, n_{+}\right)$respectively, one has

$$
\begin{aligned}
W_{1}\left(\bar{\mu} \delta_{0}+\bar{n} \mid \mu_{+} \delta_{0}+n_{+}\right) & =\int_{0}^{\infty}\left|\bar{N}-N_{+}\right| \mathrm{d} x=\int_{0}^{\infty}\left(\bar{N}-N_{+}\right) \mathrm{d} x \\
& =\int_{0}^{\infty} x\left(n_{+}-\bar{n}\right) \mathrm{d} x,
\end{aligned}
$$

recalling that $n_{+}$does not depend on time, we deduce that,

$$
\begin{aligned}
\frac{\mathrm{d}}{\mathrm{d} t} W_{1}\left(\bar{\mu} \delta_{0}+\bar{n} \mid \mu_{+} \delta_{0}+n_{+}\right) & =-\frac{\mathrm{d}}{\mathrm{d} t} \int_{0}^{\infty} x \bar{n} \mathrm{~d} x \\
& =\int_{0}^{\infty}\left(\partial_{x} \bar{n}+\chi \bar{\mu}^{2} n\right) \mathrm{d} x \\
& =-\bar{n}(t, 0)+\chi \bar{\mu}^{2}(1-\bar{\mu}) \\
& =-\frac{\mathrm{d}}{\mathrm{d} t} \bar{\mu}-\bar{\mu} P(\bar{\mu}),
\end{aligned}
$$

and

$$
\begin{aligned}
\frac{\mathrm{d}}{\mathrm{d} t}\left(\bar{\mu}-\mu_{+}+W_{1}\left(\bar{\mu} \delta_{0}+\bar{n} \mid \mu_{+} \delta_{0}+n_{+}\right)\right) & =-\bar{\mu} P(\bar{\mu}) \\
& \leq-\mu_{+} P^{\prime}\left(\mu_{+}\right)\left(\bar{\mu}-\mu_{+}\right) \leq 0,
\end{aligned}
$$

by the convexity of $P$ and $P\left(\mu_{+}\right)=0$. In particular, we deduce that

$$
\int_{0}^{\infty}\left|\bar{\mu}(t)-\mu_{+}\right| \mathrm{d} t \leq \frac{1}{\mu_{+} P^{\prime}\left(\mu_{+}\right)}\left(\int_{0}^{\infty}\left|N_{\bar{\mu}_{0}}(x)-N_{+}(x)\right| \mathrm{d} x+\bar{\mu}_{0}-\mu_{+}\right)<+\infty,
$$

which is the first statement in the Lemma. In order to proceed with the latter estimates (25)- (26), we shall re-use the entropy part of the error estimate $(23)$. Indeed, this provides some information about the first order moments, as stated in the following intermediate result.

Lemma 4.5. Let $p$ be a probability distribution on $\mathbb{R}_{+}$such that for some $\lambda>0$, the following relative entropy is finite:

$$
\mathcal{H}\left(p \mid \lambda e^{-\lambda x}\right)=\int_{0}^{\infty} p \log \frac{p}{\lambda e^{-\lambda x}} \mathrm{~d} x<+\infty .
$$

Denote $J=\int_{0}^{\infty} x p \mathrm{~d} x$. It is finite and is controlled as follows:

$$
\mathcal{H}\left(p \mid \lambda e^{-\lambda x}\right) \geq \mathcal{H}\left(p \mid \frac{1}{J} e^{-\frac{x}{J}}\right)+\frac{\left(J-\lambda^{-1}\right)^{2}}{2\left(\lambda^{-2}+J^{2}\right)} .
$$

Proof of Lemma 4.5. A straightforward computation gives

$$
\begin{aligned}
\mathcal{H}\left(p \mid \lambda e^{-\lambda x}\right) & =\mathcal{H}\left(p \mid \frac{1}{J} e^{-\frac{x}{J}}\right)+\log \frac{1}{J \lambda}+\left(\lambda-\frac{1}{J}\right) \int_{0}^{\infty} x p \mathrm{~d} x \\
& \geq \mathcal{H}\left(p \mid \frac{1}{J} e^{-\frac{x}{J}}\right)+\lambda J-1-\log (\lambda J)
\end{aligned}
$$


and the result follows from a Taylor expansion

$$
z-1-\log z \geq \frac{(z-1)^{2}}{2 \max \left(1, z^{2}\right)} \geq \frac{(z-1)^{2}}{2\left(1+z^{2}\right)} .
$$

Keeping the notation $\bar{m}=(1-\bar{\mu}) \bar{n}$, as a direct application of Lemma 4.5] and 23], together with the a priori bound $(22)$, we obtain that there is a constant $C$ such that

$$
\left(\int_{0}^{\infty} x \bar{m} \mathrm{~d} x-\frac{1}{\chi \mu_{+}^{2}}\right)^{2} \leq C \int_{0}^{\infty} \bar{n} \log \frac{\bar{m}}{m_{+}} \mathrm{d} x \leq \frac{C}{1+t} .
$$

This enables to improve the previous estimation [28):

$$
\begin{aligned}
W_{1}\left(\bar{\mu} \delta_{0}+\bar{n} \mid \mu_{+} \delta_{0}+n_{+}\right) & \leq\left(1-\mu_{+}\right) \int_{0}^{\infty} x m_{+} \mathrm{d} x-(1-\bar{\mu}) \int_{0}^{\infty} x \bar{m} \mathrm{~d} x \\
& \leq\left(\bar{\mu}-\mu_{+}\right) \int_{0}^{\infty} x m_{+} \mathrm{d} x+(1-\bar{\mu})\left(\int_{0}^{\infty} x m_{+} \mathrm{d} x-\int_{0}^{\infty} x \bar{m} \mathrm{~d} x\right) \\
& \leq \frac{C}{\sqrt{1+t}},
\end{aligned}
$$

where we have also used the error estimate $\left|\bar{\mu}-\mu_{+}\right|=O\left((1+t)^{-1 / 2}\right)$. Finally, integrating $(29)$ between $t$ and $+\infty$, it follows that

$$
\mu_{+} P^{\prime}\left(\mu_{+}\right) \int_{t}^{\infty}\left(\bar{\mu}-\mu_{+}\right) \mathrm{d} s \leq W_{1}\left(\bar{\mu} \delta_{0}+\bar{n} \mid \mu_{+} \delta_{0}+n_{+}\right)+\bar{\mu}-\mu_{+} \leq \frac{C}{\sqrt{1+t}},
$$

which ends the proof of the Lemma 4.4 .

Corollary 4.6. Under the same assumptions as in Theorem 1.2. one has

$$
\forall p \geq 1, \quad \int_{t}^{\infty}|\bar{\mu}(s)-\underline{\mu}(s)|^{p} \mathrm{~d} s \leq \frac{C_{p}}{(1+t)^{p / 2}},
$$

and

$$
\int_{0}^{t}(1+s)|\bar{\mu}(s)-\underline{\mu}(s)|^{2} \mathrm{~d} s \leq C(1+\log (1+t)) .
$$

Proof of Corollary 4.6. From corollary 4.3 we already have $\sup _{s \geq t}|\bar{\mu}(s)-\underline{\mu}(s)|^{2} \leq \frac{C}{1+t}$. We compute

$$
\int_{t}^{\infty}|\bar{\mu}-\underline{\mu}|^{p} \mathrm{~d} s \leq \sup _{s \geq t}|\bar{\mu}-\underline{\mu}|^{p-1} \int_{t}^{\infty}|\bar{\mu}-\underline{\mu}| \mathrm{d} s \leq \frac{C_{p}}{(1+t)^{p / 2}} .
$$

Additionnaly, we have

$$
\begin{aligned}
\int_{0}^{t}(1+s)|\bar{\mu}-\underline{\mu}|^{2} \mathrm{~d} s & =\left[-(1+s) \int_{s}^{\infty}|\bar{\mu}-\underline{\mu}|^{2} \mathrm{~d} y\right]_{0}^{t}+\int_{0}^{t} \int_{s}^{\infty}|\bar{\mu}-\underline{\mu}|^{2} \mathrm{~d} y \mathrm{~d} s \\
& \leq C+C \int_{0}^{t} \frac{\mathrm{d} s}{1+s}
\end{aligned}
$$

leading to the result. 


\subsection{The nonsymmetric case}

We are now ready to prove Theorem 1.2 without the symmetry hypothesis. As a direct application of the comparison principle, $N(t, \cdot) \rightarrow N_{+}$in $L^{1}$ norm (i.e. in $W_{1}$ distance between $n$ and $n_{+}$). This can be reinforced to the convergence $n(t, \cdot) \rightarrow n_{+}$in $L^{1}$ norm using the relative entropy, as in the symmetric problem.

As before, we introduce the functional $L_{i}$ and the dissipation $D_{i}$ by

$$
L_{i}(t)=\left(1-\mu_{i}\right) \mathcal{H}\left(m_{i} \mid m_{+}\right), \quad D_{i}^{2}=\left(1-\mu_{i}\right) \mathcal{I}\left(m_{i} \mid \chi \mu_{1} \mu_{2} e^{-\chi \mu_{1} \mu_{2} x}\right)
$$

Performing similar computations as in Section 4.1, we obtain

$$
\begin{aligned}
\frac{\mathrm{d}}{\mathrm{d} t}\left(L_{1}+L_{2}\right) & =\sum_{i=1,2}\left(-D_{i}^{2}+\left(\log \frac{\mu_{i}}{n_{i}(t, 0)}\right) \frac{\mathrm{d} \mu_{i}}{\mathrm{~d} t}+\left(\log \frac{\left(1-\mu_{i}\right) m_{+}(0)}{\mu_{i}}\right) \frac{\mathrm{d} \mu_{i}}{\mathrm{~d} t}\right. \\
& \left.+\chi\left(\mu_{1} \mu_{2}-\mu_{+}^{2}\right)\left(-n_{i}(t, 0)+\chi \mu_{1} \mu_{2}\left(1-\mu_{i}\right)\right)\right) .
\end{aligned}
$$

Let the function $f_{\log }$ be defined by

$$
f_{\log }\left(\mu_{+}\right)=0, \quad f_{\log }^{\prime}(\mu)=\log \frac{\mu}{(1-\mu) m_{+}(0)}=\log \frac{\mu\left(1-\mu_{+}\right)}{(1-\mu) \mu_{+}}
$$

We observe that $f_{\log }^{\prime}\left(\mu_{+}\right)=0$ and the second derivative satisfies $f_{\log }^{\prime \prime}(\mu)>0$ for $\mu \in(0,1)$. Hence, locally in the neighborhood of $\mu_{+}$, the function $f_{\log }$ behaves as $\left(\mu-\mu_{+}\right)^{2}$.

Following (31), using that $\mu_{1} \mu_{2}=\left(\frac{\mu_{1}+\mu_{2}}{2}\right)^{2}-\left(\frac{\mu_{1}-\mu_{2}}{2}\right)^{2}$ and recalling that $\frac{\mathrm{d}}{\mathrm{d} t} \mu_{i}=n_{i}(t, 0)-\mu_{i}$, we obtain

$$
\begin{aligned}
\chi\left(\mu_{1} \mu_{2}-\mu_{+}^{2}\right)\left(n_{1}(t, 0)+n_{2}(t, 0)\right)= & 2 \frac{\mathrm{d}}{\mathrm{d} t}\left(g\left(\frac{\mu_{1}+\mu_{2}}{2}\right)\right) \\
& +\chi\left(\left(\frac{\mu_{1}+\mu_{2}}{2}\right)^{2}-\mu_{+}^{2}\right)\left(\mu_{1}+\mu_{2}\right)-\chi \frac{\left(\mu_{1}-\mu_{2}\right)^{2}}{4}\left(n_{1}(t, 0)+n_{2}(t, 0)\right),
\end{aligned}
$$

where the function $g$ is defined by

$$
g(\mu)=\chi\left(\frac{\mu^{3}}{3}-\mu_{+}^{2} \mu+2 \frac{\mu_{+}^{3}}{3}\right)=\chi \frac{\left(\mu-\mu_{+}\right)^{2}\left(\mu+2 \mu_{+}\right)}{3}, \quad g^{\prime}(\mu)=\chi\left(\mu^{2}-\mu_{+}^{2}\right) .
$$

We combine these contributions into the following functional $\widetilde{L}$ :

$$
\widetilde{L}(t)=L_{1}(t)+L_{2}(t)+f_{\log }\left(\mu_{1}(t)\right)+f_{\log }\left(\mu_{2}(t)\right)+2 g\left(\frac{\mu_{1}(t)+\mu_{2}(t)}{2}\right) .
$$

From the comparison principle and the HWI inequality, it follows that there exists a positive constant $C$ such that $D_{1}^{2}+D_{2}^{2} \geq C\left(L_{1}^{2}+L_{2}^{2}\right)$, as in the proof of Lemma 4.2 - estimate 21. Hence the dissipation estimate (31) becomes

$$
\frac{\mathrm{d} \widetilde{L}}{\mathrm{~d} t}(t) \leq-C\left(L_{1}(t)^{2}+L_{2}(t)^{2}\right)+h\left(\mu_{1}(t), \mu_{2}(t)\right)+R(t)
$$


where

$$
\begin{aligned}
R(t)=-\log \frac{n_{1}(t, 0)}{\mu_{1}}\left(n_{1}(t, 0)-\mu_{1}\right)-\log \frac{n_{2}(t, 0)}{\mu_{2}}\left(n_{2}(t, 0)-\mu_{2}\right) & \\
& +\chi \frac{\left(\mu_{1}-\mu_{2}\right)^{2}}{4}\left(n_{1}(t, 0)+n_{2}(t, 0)\right),
\end{aligned}
$$

and $h$ is defined by

$$
h\left(\mu_{1}, \mu_{2}\right)=\chi^{2} \mu_{1} \mu_{2}\left(\mu_{1} \mu_{2}-\mu_{+}^{2}\right)\left(2-\mu_{1}-\mu_{2}\right)-\chi\left(\left(\frac{\mu_{1}+\mu_{2}}{2}\right)^{2}-\mu_{+}^{2}\right)\left(\mu_{1}+\mu_{2}\right) .
$$

Since $h\left(\mu_{+}, \mu_{+}\right)=0, \nabla h\left(\mu_{+}, \mu_{+}\right)=0$, and the matrix $\left.\nabla^{2} h\right|_{\left(\mu_{+}, \mu_{+}\right)}$is symmetric definite negative, then, locally in the neighborhood of $\left(\mu_{+}, \mu_{+}\right)$, there exists a positive constant $C$ such that

$$
h\left(\mu_{1}, \mu_{2}\right) \leq-C\left(\left(\mu_{1}-\mu_{+}\right)^{2}+\left(\mu_{2}-\mu_{+}\right)^{2}\right) \leq-C\left(f_{\log }\left(\mu_{1}(t)\right)+f_{\log }\left(\mu_{2}(t)\right)\right) .
$$

Therefore, for $t \geq t_{0}$ large enough, so that the $\mu_{i}$ are close enough to $\mu_{+}$, up to a change of the value of the constant $C>0$, we have

$$
\frac{\mathrm{d}}{\mathrm{d} t} \widetilde{L}(t) \leq-C \widetilde{L}(t)^{2}+R(t)
$$

Note that in the symmetric case, it is possible to conclude from this stage, as done previously. However, in the general case, the additional contribution involving $\left(\mu_{1}-\mu_{2}\right)^{2}$ in $\sqrt{32}$ requires more work to handle with. It follows from inequality (33) that $\widetilde{L}$ is bounded. Indeed, denoting $F(t)=(1+t) \widetilde{L}(t)$, we see that the previous inequality (33) rewrites as

$$
F^{\prime}(t) \leq \widetilde{L}(t)-C(1+t) \widetilde{L}(t)^{2}+(1+t) R(t)
$$

from which we deduce that

$$
F^{\prime}(t) \leq \frac{1}{1+t}\left(F-C F^{2}\right)+(1+t) R(t) .
$$

We first notice the obvious upper bound $F-C F^{2} \leq(4 C)^{-1}$, so that we have

$$
F(t) \leq F(0)+\frac{1}{4 C} \log (1+t)+\int_{0}^{t}(1+s) R(s) \mathrm{d} s .
$$

In order to estimate the last term, we observe that $R=R_{1}+R_{2}$, where

$$
\begin{aligned}
R_{i}(t) & :=-\log \frac{n_{i}(t, 0)}{\mu_{i}}\left(n_{i}(t, 0)-\mu_{i}\right)+\chi \frac{\left(\mu_{1}-\mu_{2}\right)^{2}}{4} n_{i}(t, 0) \\
& =\left(n_{i}(t, 0)-\mu_{i}\right)\left(-\log \frac{n_{i}(t, 0)}{\mu_{i}}+\chi \frac{\left(\mu_{1}-\mu_{2}\right)^{2}}{4}\right)+\chi \frac{\left(\mu_{1}-\mu_{2}\right)^{2}}{4} \mu_{i} .
\end{aligned}
$$

The first contribution in the right hand-side of the previous equality is nonnegative if and only if

$$
\mu_{i} \leq n_{i}(t, 0) \leq \mu_{i} e^{\chi \frac{\left(\mu_{1}-\mu_{2}\right)^{2}}{4}} .
$$

Moreover, we see that

$$
R_{i}(t) \leq \chi \mu_{i} \frac{\left(\mu_{1}-\mu_{2}\right)^{2}}{4}\left(e^{\chi \frac{\left(\mu_{1}-\mu_{2}\right)^{2}}{4}}-1\right)+\chi \frac{\left(\mu_{1}-\mu_{2}\right)^{2}}{4} \mu_{i} .
$$


Then, using the mean value theorem and adding $R_{1}+R_{2}$, we have

$$
R(t) \leq C\left(\mu_{1}-\mu_{2}\right)^{2} \leq C(\bar{\mu}-\underline{\mu})^{2},
$$

by the comparison principle, where $\bar{\mu}$ and $\underline{\mu}$ are defined as in Section 4.2 . Hence recalling (30), we deduce that

$$
\int_{0}^{t}(1+s) R(s) \mathrm{d} s \leq C(1+\log (1+t)) .
$$

So that, we finally obtain

$$
F(t)-F(0) \leq C(1+\log (1+t))
$$

and therefore

$$
\widetilde{L}(t) \leq C \frac{1+\log (1+t)}{(1+t)} .
$$

The statements of Theorem 1.2 follow from this entropy estimate as in Corollary 4.3

\section{Self-Similar decay: Proof of Theorem 1.3}

As in the previous section, we consider successively the symmetric case and the general case.

\subsection{Self-similar decay in the symmetric case}

It is natural to perform the following parabolic rescaling in order to catch the self-similar behaviour:

$$
n(t, x)=\frac{1}{\sqrt{1+2 t}} u\left(\frac{1}{2} \log (1+2 t), \frac{x}{\sqrt{1+2 t}}\right)=\frac{1}{\sqrt{1+2 t}} u(\tau, y)
$$

which is mass-preserving

$$
\int_{0}^{\infty} u(\tau, y) \mathrm{d} y=\int_{0}^{\infty} n\left(\frac{e^{2 \tau}-1}{2}, x\right) \mathrm{d} x=1-\mu\left(\frac{e^{2 \tau}-1}{2}\right)=1-\nu(\tau),
$$

where we have set $\mu(t)=\mu\left(\frac{e^{2 \tau}-1}{2}\right)=\nu(\tau)$. Since $(n, \mu)$ is solution to 16, $(u, \nu)$ satisfies the following boundary value problem:

$$
\left\{\begin{array}{l}
\partial_{\tau} u(\tau, y)-\partial_{y y} u(\tau, y)-\partial_{y}(y u(\tau, y))-\chi \nu(\tau)^{2} e^{\tau} \partial_{y} u(\tau, y)=0, \quad(\tau, y) \in(0, \infty)^{2} \\
\frac{\mathrm{d} \nu}{\mathrm{d} \tau}(\tau)=\partial_{y} u(\tau, 0)+\chi \nu(\tau)^{2} e^{\tau} u(\tau, 0)=e^{\tau} u(\tau, 0)-e^{2 \tau} \nu(\tau)
\end{array}\right.
$$

with the same initial data as in the original variables. Note that the additional left-sided drift $\partial_{y}(y u(\tau, y))$ contributes to confine the mass in the new frame $(\tau, y)$.

Then, Theorem 1.3 is a consequence of the following formulation in self-similar variables (in the symmetric case):

Proposition 5.1. Under the same assumptions as in Proposition 1.3, the following convergence estimates hold true:

$$
\nu(\tau)+\int_{0}^{\infty} u \log \frac{u}{(1-\nu) G} \leq C e^{-\tau} .
$$

In particular, it follows from the Csiszar-Kullbáck inequality that

$$
\|u-G\|_{1} \leq C e^{-\frac{\tau}{2}} .
$$


Proof of Proposition 5.1. We split the proof into several intermediate results. We start by establishing bounds on the first and second moments and then we prove entropy dissipation.

Lemma 5.2. There exist two constants $M_{1}$ and $M_{2}$ depending only on $\int_{0}^{\infty} y^{2} u(0, y) \mathrm{d} y$ such that

$$
\forall \tau>0, \quad \int_{0}^{\infty} y u(\tau, y) \mathrm{d} y \leq M_{1} \quad \text { and } \quad \int_{0}^{\infty} y^{2} u(\tau, y) \mathrm{d} y \leq M_{2} .
$$

Proof of Lemma 5.2. We first see that

$$
\begin{aligned}
\frac{\mathrm{d}}{\mathrm{d} \tau} \int_{0}^{\infty} y^{2} u(\tau, y) \mathrm{d} y & =2(1-\nu(\tau))-2 \chi \nu(\tau)^{2} e^{\tau} \int_{0}^{\infty} y u(\tau, y) \mathrm{d} y-2 \int_{0}^{\infty} y^{2} u(\tau, y) \mathrm{d} y, \\
& \leq 2-2 \int_{0}^{\infty} y^{2} u(\tau, y) \mathrm{d} y
\end{aligned}
$$

from which it follows that

$$
\int_{0}^{\infty} y^{2} u(\tau, y) \mathrm{d} y \leq M_{2}=\max \left(1, \int_{0}^{\infty} y^{2} u(0, y) \mathrm{d} y\right) .
$$

Using the Cauchy-Schwarz inequality, we deduce that

$$
\int_{0}^{\infty} y u(\tau, y) \mathrm{d} y \leq \sqrt{1-\nu(\tau)} \sqrt{M_{2}} \leq \sqrt{M_{2}}=: M_{1} .
$$

Let us now prove entropy dissipation. To do so, we compare the solution $u$ to the normalized gaussian $G$ on the half line. We begin with some notations. Let the renormalized density $v$ such that $u(\tau, y)=(1-\nu(\tau)) v(\tau, y)$. Consider the following functional $\mathcal{L}$ :

$$
\mathcal{L}(\tau)=\underbrace{(1-\nu(\tau)) \mathcal{H}(v(\tau, \cdot) \mid G)}_{\mathcal{L}_{1}}+\underbrace{H(1-\nu(\tau))}_{\mathcal{L}_{2}}+\underbrace{G(0) e^{-\tau} H\left(\frac{\nu(\tau)}{G(0) e^{-\tau}}\right)}_{\mathcal{L}_{3}}+\underbrace{\frac{\chi}{3} \nu(\tau)^{3}}_{\mathcal{L}_{4}},
$$

where $H(x)=x \log x-x+1$ and where we have defined various contributions $\mathcal{L}_{i}$ that will be studied separately. Since $\mathcal{H}$ and $H$ is non-negative, $\mathcal{L}$ is also non-negative. Notice that $\mathcal{L}$ can be written alternatively as

$$
\mathcal{L}(\tau)=\int_{0}^{\infty} u(\tau, y) \log \frac{u(\tau, y)}{G(y)} \mathrm{d} y+\nu(\tau) \log \frac{\nu(\tau)}{G(0) e^{-\tau}}+G(0) e^{-\tau}+\frac{\chi}{3} \nu(\tau)^{3} .
$$

Finally, let introduce the following auxiliary function that will play a pivotal role:

$$
G_{\nu ; \tau}(y)=\frac{e^{-\chi \nu^{2} e^{\tau} y-\frac{y^{2}}{2}}}{\int_{0}^{\infty} e^{-\chi \nu^{2} e^{\tau} z-\frac{z^{2}}{2}} \mathrm{~d} z} .
$$

Also, recall the definition of the Fisher information:

$$
\mathcal{I}\left(u \mid G_{\nu(\tau) ; \tau}\right)=\int_{0}^{\infty} u(\tau, y)\left(\partial_{y} \log u(\tau, y)+y+\chi \nu(\tau)^{2} e^{\tau}\right)^{2} \mathrm{~d} y \geq 0 .
$$

In the following Lemma we obtain upper bounds on $\frac{\mathrm{d} \mathcal{L}}{\mathrm{d} \tau}(\tau)$. 
Lemma 5.3. The following upper bound holds true:

$$
\begin{aligned}
\frac{\mathrm{d} \mathcal{L}}{\mathrm{d} \tau}(\tau) \leq- & \mathcal{I}\left(u \mid G_{\nu(\tau) ; \tau}\right)+\nu(\tau)-G(0) e^{-\tau} \\
& +\chi \nu(\tau)^{2} e^{\tau} \int_{0}^{\infty} y u(\tau, y) \mathrm{d} y-\chi \nu(\tau)^{3} e^{2 \tau} P(\nu(\tau))
\end{aligned}
$$

where the polynomial function $P$ is defined by (7).

Proof of lemma 5.3. Differentiating 35, we obtain

$$
\begin{aligned}
\frac{\mathrm{d} \mathcal{L}}{\mathrm{d} \tau}(\tau) & =\int_{0}^{\infty} \log \frac{u(\tau, y)}{G(y)} \partial_{\tau} u(\tau, y) \mathrm{d} y+\left(\log \frac{\nu(\tau)}{G(0) e^{-\tau}}+\chi \nu(\tau)^{2}\right) \frac{\mathrm{d} \nu}{\mathrm{d} \tau}(\tau)+\nu(\tau)-G(0) e^{-\tau} \\
& =-\left(\log \frac{u(\tau, 0)}{G(0)}\right) \frac{\mathrm{d} \nu}{\mathrm{d} \tau}(\tau)+\left(\log \frac{\nu(\tau)}{G(0) e^{-\tau}}+\chi \nu(\tau)^{2}\right) \frac{\mathrm{d} \nu}{\mathrm{d} \tau}(\tau)+\nu(\tau)-G(0) e^{-\tau} \\
& -\int_{0}^{\infty} u(\tau, y)\left(\partial_{y} \log u(\tau, y)+y\right)\left(\partial_{y} \log u(\tau, y)+y+\chi \nu(\tau)^{2} e^{\tau}\right) \mathrm{d} y \\
& =-\left(\log \frac{u(\tau, 0)}{\nu(\tau) e^{\tau}}\right) \frac{\mathrm{d} \nu}{\mathrm{d} \tau}(\tau)+\nu(\tau)-G(0) e^{-\tau}+\chi \nu(\tau)^{2} e^{\tau} u(\tau, 0)-\chi \nu(\tau)^{3} e^{2 \tau} \\
& -\int_{0}^{\infty} u(\tau, y)\left(\partial_{y} \log u(\tau, y)+y\right)\left(\partial_{y} \log u(\tau, y)+y+\chi \nu(\tau)^{2} e^{\tau}\right) \mathrm{d} y
\end{aligned}
$$

Using the definition 37 of $\mathcal{I}\left(u \mid G_{\nu(\tau) ; \tau}\right)$, we have

$$
\begin{aligned}
\frac{\mathrm{d} \mathcal{L}}{\mathrm{d} \tau}(\tau) \leq- & \mathcal{I}\left(u \mid G_{\nu(\tau) ; \tau}\right)+\nu(\tau)-G(0) e^{-\tau}+\chi \nu(\tau)^{2} e^{\tau} u(\tau, 0)-\chi \nu(\tau)^{3} e^{2 \tau} \\
& \quad+\chi \nu(\tau)^{2} e^{\tau} \int_{0}^{\infty} u(\tau, y)\left(\partial_{y} \log u(\tau, y)+y+\chi \nu(\tau)^{2} e^{\tau}\right) \mathrm{d} y \\
\leq & -\mathcal{I}\left(u \mid G_{\nu(\tau) ; \tau}\right)+\nu(\tau)-G(0) e^{-\tau} \\
& \quad+\chi \nu(\tau)^{2} e^{\tau} \int_{0}^{\infty} y u(\tau, y) \mathrm{d} y+\left(\chi \nu(\tau)^{2} e^{\tau}\right)^{2}(1-\nu(\tau))-\chi \nu(\tau)^{3} e^{2 \tau} \\
\leq- & \mathcal{I}\left(u \mid G_{\nu(\tau) ; \tau}\right)+\nu(\tau)-G(0) e^{-\tau} \\
& +\chi \nu(\tau)^{2} e^{\tau} \int_{0}^{\infty} y u(\tau, y) \mathrm{d} y-\chi \nu(\tau)^{3} e^{2 \tau} P(\nu(\tau)),
\end{aligned}
$$

which is the estimate 38 .

Several lower bound on the relative entropy are required to control entropy dissipation.

Lemma 5.4. The following inequality holds true

$$
\mathcal{I}\left(u \mid G_{\nu ; \tau}\right) \geq 2 \mathcal{L}_{1}-2 \chi \nu^{2} e^{\tau} \int_{0}^{\infty} y((1-\nu) G-u) \mathrm{d} y .
$$

Proof of Lemma 5.4. Recalling the definition (36) of $G_{\nu ; \tau}$, the logarithmic Sobolev inequality (Lemma B.5) yields that

$$
\mathcal{I}\left(u \mid G_{\nu ; \tau}\right) \geq 2 \int_{0}^{\infty} u \log \frac{u}{(1-\nu) G_{\nu ; \tau}} \mathrm{d} y .
$$


The latter term can be decomposed as follows,

$$
\int_{0}^{\infty} u \log \frac{u}{(1-\nu) G_{\nu ; \tau}} \mathrm{d} y=\underbrace{\int_{0}^{\infty} u \log \frac{u}{(1-\nu) G} \mathrm{~d} y}_{\mathcal{L}_{1}}+\int_{0}^{\infty} u \log \frac{G}{G_{\nu ; \tau}} \mathrm{d} y .
$$

Using Jensen's inequality, we have

$$
\begin{aligned}
\log \frac{G}{G_{\nu ; \tau}} & =\chi \nu^{2} e^{\tau} y+\log \frac{\int_{0}^{\infty} e^{-\chi \nu^{2} e^{\tau} z-\frac{z^{2}}{2}} \mathrm{~d} z}{\int_{0}^{\infty} e^{-\frac{z^{2}}{2}} \mathrm{~d} z} \\
& \geq \chi \nu^{2} e^{\tau} y-\int_{0}^{\infty} \chi \nu^{2} e^{\tau} z G(z) \mathrm{d} z .
\end{aligned}
$$

This completes the proof of Lemma 5.4 .

We can now derive a quantitative rate of convergence.

Lemma 5.5. There exists a positive constant $C$ such that

$$
\mathcal{L}(\tau) \leq C e^{-\tau} .
$$

Proof of Lemma 5.5. Injecting the result of Lemma 5.4 into inequality (38), it follows that

$$
\begin{aligned}
\frac{\mathrm{d} \mathcal{L}}{\mathrm{d} \tau}(\tau) \leq & -2 \mathcal{L}_{1}+\nu-G(0) e^{-\tau}-\chi \nu^{2} e^{\tau} \int_{0}^{\infty} y u \mathrm{~d} y \\
& +2 \chi \nu^{2} e^{\tau} \int_{0}^{\infty} y(1-\nu) G \mathrm{~d} y-\chi \nu^{3} e^{2 \tau} P(\nu) \\
\leq & -2 \mathcal{L}_{1}(\tau)+\nu-G(0) e^{-\tau}+2 \chi \nu^{2} e^{\tau}(1-\nu) G(0)-\chi \nu^{3} e^{2 \tau} P(\nu) .
\end{aligned}
$$

Under the assumptions of Proposition 5.1. there exists $P_{0}>0$ such that $P(\nu(\tau)) \geq P_{0}$ for all $\tau \geq 0$. Indeed, either $\chi \geq 4$ and $P(\mu(t)) \geq P\left(\mu_{0}\right)$ by the comparison principle (Lemma 3.2), or $\chi<4$ and $P$ is uniformly bounded from below.

To conclude, let add $2 \mathcal{L}$ on both sides in order to get

$$
\frac{\mathrm{d} \mathcal{L}}{\mathrm{d} \tau}(\tau)+2 \mathcal{L}(\tau) \leq \nu-G(0) e^{-\tau}-\chi \nu^{3} e^{2 \tau} P_{0}+2 \chi G(0) \nu^{2} e^{\tau}+2 \mathcal{L}_{2}(\tau)+2 \mathcal{L}_{3}(\tau)+2 \mathcal{L}_{4}(\tau) .
$$

From the definitions of $\mathcal{L}_{2}, \mathcal{L}_{3}$ and $\mathcal{L}_{4}$ (34), we see that

$$
\begin{aligned}
& \mathcal{L}_{2}(\tau) e^{2 \tau}=((1-\nu) \log (1-\nu)+\nu) e^{2 \tau} \leq \nu e^{2 \tau}, \\
& \mathcal{L}_{3}(\tau) e^{2 \tau}=G(0) e^{\tau} H\left(\frac{\nu e^{\tau}}{G(0)}\right)=\nu e^{2 \tau} \log \frac{\nu e^{\tau}}{G(0)}-\nu e^{2 \tau}+G(0) e^{\tau} \\
& \mathcal{L}_{4}(\tau) e^{2 \tau}=\frac{\chi}{3} \nu^{3} e^{2 \tau} \leq \frac{\chi}{3} \nu e^{2 \tau} .
\end{aligned}
$$

Consequently, we obtain

where the function $h$ is defined by

$$
\frac{\mathrm{d}}{\mathrm{d} \tau}\left(e^{2 \tau} \mathcal{L}(\tau)\right) \leq e^{\tau} h\left(\nu e^{\tau}\right)
$$

$$
h(z)=-\chi P_{0} z^{3}+2 \chi G(0) z^{2}+2 z \log \frac{z}{G(0)}+G(0)+z+\frac{\chi}{3} z .
$$


The function $h$ is clearly bounded from above. Therefore, after integration between 0 and $\tau$, we obtain

$$
\begin{aligned}
& \mathcal{L}(\tau) e^{2 \tau} \leq \mathcal{L}(0)+e^{\tau} \sup h, \\
& \mathcal{L}(\tau) \leq(\mathcal{L}(0)+\sup h) e^{-\tau} .
\end{aligned}
$$

This concludes the proofs of Lemma 5.5 and Proposition 5.1.

\subsection{Self-similar decay in the nonsymmetric case}

In the general case, the solution can be bounded from above by the special solution of the symmetric problem initialized by $\left(N_{\mu_{0}}, N_{\mu_{0}}\right)$, due to the comparison principle (Lemma 3.2 and 3.3). We deduce the following rate of convergence:

$$
\nu_{i}(\tau) \leq C e^{-\tau}, \quad i=1,2 .
$$

With similar notations as before, we define the functionals $\mathcal{L}_{\text {gen }}^{(i)}$ by

$$
\mathcal{L}_{\text {gen }}^{(i)}(\tau)=\mathcal{L}_{1}^{(i)}+\mathcal{L}_{2}^{(i)}+\mathcal{L}_{3}^{(i)}, \quad i=1,2,
$$

where each $\mathcal{L}_{j}^{(i)}$ is defined as in (34), but involving $u_{i}, \nu_{i}$. An important point here is that it does not contain the correction term $\mathcal{L}_{4}$. Following the previous computations, we obtain

$$
\begin{aligned}
\frac{\mathrm{d}}{\mathrm{d} \tau} \mathcal{L}_{\text {gen }}^{(i)}(\tau) \leq- & \mathcal{I}^{(i)}+\nu_{i}-G(0) e^{-\tau}-\chi \nu_{1} \nu_{2} e^{\tau} u_{i}(\tau, 0)+\chi \nu_{i} \nu_{1} \nu_{2} e^{2 \tau} \\
& +\chi \nu_{i}^{2} e^{\tau} \int_{0}^{\infty} y u_{i}(\tau, y) \mathrm{d} y-\chi \nu_{i} \nu_{1} \nu_{2} e^{2 \tau} P\left(\nu_{i}(\tau)\right)
\end{aligned}
$$

where we have denoted

$$
\mathcal{I}^{(i)}=\int_{0}^{\infty} u_{i}\left(\partial_{y} \log y+y+\chi \nu_{1} \nu_{2} e^{\tau}\right)^{2} \mathrm{~d} y .
$$

Using the estimate $\nu_{i} \leq C e^{-\tau}$, this leads to

$$
\frac{\mathrm{d}}{\mathrm{d} \tau} \mathcal{L}_{\text {gen }}^{(i)}(\tau) \leq-\mathcal{I}^{(i)}+C e^{-\tau} .
$$

Using similar computations as before, we obtain

$$
\mathcal{L}_{\text {gen }}^{(i)}(\tau) \leq C e^{-\tau} .
$$

This concludes the proof of Theorem 1.3 .

\section{REFERENCES}

[1] Bruce Alberts, Alexander Johnson, Julian Lewis, Martin Raff, Keith Roberts, and Peter Walter. Molecular Biology of the Cell. Garland Science, 5th edition, 2007.

[2] Anton Arnold, Peter Markowich, Giuseppe Toscani, and Andreas Unterreiter. On convex sobolev inequalities and the rate of convergence to equilibrium for Fokker-Planck type equations. Communications in Partial Differential Equations, 26(1-2):43$100,2001$.

[3] Michel Bagnat and Kai Simons. Cell surface polarization during yeast mating. Proceedings of the National Academy of Sciences, 99(22):14183-14188, 2002.

[4] Piotr Biler, Grzegorz Karch, Philippe Laurençot, and Tadeusz Nadzieja. The $8 \pi$-problem for radially symmetric solutions of a chemotaxis model in the plane. Mathematical Methods in the Applied Sciences, 29(13):1563-1583, 2006.

[5] Adrien Blanchet. On the parabolic-elliptic Patlak-Keller-Segel system in dimension 2 and higher. Séminaire Laurent Schwartz - EDP et applications, 2011-2012. 
[6] María J Cáceres, José A Carrillo, and Benoît Perthame. Analysis of nonlinear noisy integrate \& fire neuron models: blow-up and steady states. The Journal of Mathematical Neuroscience, 1(1):7, 2011.

[7] María J Cáceres and Ricarda Schneider. Blow-up, steady states and long time behaviour of excitatory-inhibitory nonlinear neuron models. Kinetic 6 Related Models, 10(3), 2017.

[8] Vincent Calvez, Rhoda Hawkins, Nicolas Meunier, and Raphaël Voituriez. Analysis of a nonlocal model for spontaneous cell polarization. SIAM Journal on Applied Mathematics, 72(2):594-622, 2012.

[9] Vincent Calvez, Nicolas Meunier, and Raphaël Voituriez. A one-dimensional Keller-Segel equation with a drift issued from the boundary. C. R. Math. Acad. Sci. Paris, 348(11-12):629-634, 2010.

[10] José A Carrillo, María d M González, Maria P Gualdani, and Maria E Schonbek. Classical solutions for a nonlinear fokkerplanck equation arising in computational neuroscience. Communications in Partial Differential Equations, 38(3):385-409, 2013.

[11] José A Carrillo, Benoît Perthame, Delphine Salort, and Didier Smets. Qualitative properties of solutions for the noisy integrate and fire model in computational neuroscience. Nonlinearity, 28(9):3365, 2015.

[12] Weitao Chen, Qing Nie, Tau-Mu Yi, and Ching-Shan Chou. Modelling of yeast mating reveals robustness strategies for cell-cell interactions. PLoS Computational Biology, 12(7), 2016.

[13] Ching-Shan Chou, Lee Bardwell, Qing Nie, and Tau-Mu Yi. Noise filtering tradeoffs in spatial gradient sensing and cell polarization response. BMC Systems Biology, 5(1):196, 2011.

[14] Ching-Shan Chou, Qing Nie, and Tau-Mu Yi. Modeling robustness tradeoffs in yeast cell polarization induced by spatial gradients. PLoS ONE, 3:e3103, 2008.

[15] Imre Csiszár. Information-type measures of difference of probability distributions and indirect observations. Studia Sci. Math. Hungar., 2:299-318, 1967.

[16] Grégory Dumont, Jacques Henry, and Carmen Oana Tarniceriu. Noisy threshold in neuronal models: connections with the noisy leaky integrate-and-fire model. Journal of Mathematical Biology, 73(6):1413-1436, 2016.

[17] Jayme M Dyer, Natasha S Savage, Meng Jin, Trevin R Zyla, Timothy C Elston, and Daniel J Lew. Tracking shallow chemical gradients by actin-driven wandering of the polarization site. Current biology, 23(1):32-41, 2013.

[18] Lawrence C. Evans. Partial differential equations, volume 19 of Graduate Studies in Mathematics. American Mathematical Society, Providence, RI, 2nd edition, 2010.

[19] Tina Freisinger, Ben Klünder, Jared Johnson, Nikola Müller, Garwin Pichler, Gisela Beck, Michael Costanzo, Charles Boone, Richard A Cerione, Erwin Frey, and Roland Wedlich-Soldner. Establishment of a robust single axis of cell polarity by coupling multiple positive feedback loops. Nature communications, 4:1807, 2013.

[20] H Gajewski. On a variant of monotonicity and its application to differential equations. Nonlinear Analysis: Theory, Methods ES Applications, 22(1):73-80, 1994.

[21] Rhoda Hawkins, Olivier Bénichou, Matthieu Piel, and Raphaël Voituriez. Rebuilding cytoskeleton roads: active transport induced polarisation of cells. Phys. Rev., E 80:040903, 2009.

[22] Catherine L. Jackson and Leland H. Hartwell. Courtship in s. cerevisiae: both cell types choose mating partners by responding to the strongest pheromone signal. Cell, 63(5):1039-1051, 1990

[23] Meng Jin, Beverly Errede, Marcelo Behar, Will Mather, Sujata Nayak, Jeff Hasty, Henrik G. Dohlman, and Timothy C. Elston. Yeast dynamically modify their environment to achieve better mating efficiency. Science Signaling, 4(186):ra54-ra54, 2011.

[24] Inwon Kim and Yao Yao. The Patlak-Keller-Segel model and its variations: Properties of solutions via maximum principle. SIAM Journal on Mathematical Analysis, 44(2):568-602, 2012.

[25] Solomon Kullback. On the convergence of discrimination information. IEEE Trans. Information Theory, IT-14:765-766, 1968.

[26] Michael J. Lawson, Brian Drawert, Mustafa Khammash, Linda Petzold, and Tau-Mu Yi. Spatial stochastic dynamics enable robust cell polarization. PLoS Comput Biol, 9(7):e1003139, 072013.

[27] Anita T. Layton, Natasha S. Savage, Audrey S. Howell, Susheela Y. Carroll, David G. Drubin, and Daniel J. Lew. Modeling Vesicle Traffic Reveals Unexpected Consequences for Cdc42p-Mediated Polarity Establishment. Current Biology, 21(3):184194, 2011.

[28] Thomas Lepoutre, Nicolas Meunier, and Nicolas Muller. Cell polarisation model: the 1D case. J. Math. Pures Appl. (9), 101(2):152-171, 2014.

[29] Kevin Madden and Michael Snyder. Cell polarity and morphogenesis in budding yeast. Annual Review of Microbiology, 52(1):687-744, 1998. PMID: 9891811.

[30] Travis I Moore, Ching-Shan Chou, Qing Nie, Noo Li Jeon, and Tau-Mu Yi. Robust spatial sensing of mating pheromone gradients by yeast cells. PLoS ONE, 3:e3865, 2008.

[31] Travis I Moore, Hiromasa Tanaka, Hyung Joon Kim, Noo Li Jeon, and Tau-Mu Yi. Yeast G-proteins mediate directional sensing and polarization behaviors in response to changes in pheromone gradient direction. Molecular Biology of the Cell, 24(4):521-34, 2013.

[32] Nicolas Muller, Matthieu Piel, Vincent Calvez, Raphaël Voituriez, Joana Gonçalves-Sá, Chin-Lin Guo, Xingyu Jiang, Andrew Murray, and Nicolas Meunier. A predictive model for yeast cell polarization in pheromone gradients. PLoS Computational Biology, 12(4):e1004795, 2016. 
[33] Natasha S Savage, Anita T Layton, and Daniel J Lew. Mechanistic mathematical model of polarity in yeast. Molecular biology of the cell, 23(10):1998-2013, 2012.

[34] Marie-Noelle Simon, Claudio De Virgilio, Brian Souza, John R Pringle, Arie Abo, and Steven I Reed. Role for the rho-family gtpase cdc42 in yeast mating-pheromone signal pathway. Nature, 376(6542):702-705, 1995.

[35] Brian D Slaughter, Sarah E Smith, and Rong Li. Symmetry breaking in the life cycle of the budding yeast. Cold Spring Harbor perspectives in biology, 1(3), 2009.

[36] Brian D Slaughter, Jay R Unruh, Arupratan Das, Sarah E Smith, Boris Rubinstein, and Rong Li. Non-uniform membrane diffusion enables steady-state cell polarization via vesicular trafficking. Nature communications, 4:1380, 2013.

[37] Cédric Villani. Topics in optimal transportation, volume 58 of Graduate Studies in Mathematics. American Mathematical Society, Providence, RI, 2003.

[38] Roland Wedlich-Soldner, Steve Altschuler, Lani Wu, and Rong Li. Spontaneous cell polarization through actomyosin-based delivery of the cdc42 gtpase. Science, 299(5610):1231-1235, 2003.

[39] Roland Wedlich-Soldner, Stephanie C Wai, Thomas Schmidt, and Rong Li. Robust cell polarity is a dynamic state established by coupling transport and gtpase signaling. The Journal of cell biology, 166(6):889-900, 2004.

\section{Appendix A. Linear stability analysis: proof of Proposition 1.1}

The linear stability analysis involves a series of Lemma.

Lemma A.1. The steady states $\left(\bar{n}_{i}, \bar{\mu}_{i}\right)_{i=1,2}$ of the system (2)-(3) -(4) with (5) satisfy $\bar{n}_{i}=\bar{n}$ and $\bar{\mu}_{i}=\bar{\mu}$, for $i=1,2$, where $(\bar{n}, \bar{\mu})$ is solution to

$$
\left\{\begin{aligned}
\bar{n}(x) & =\bar{\mu} \exp \left(-\chi \bar{\mu}^{2} x\right), \quad x \geq 0, \\
0 & =\chi \bar{\mu}^{2}-\chi \bar{\mu}+1 .
\end{aligned}\right.
$$

Such a steady state exists iff $\chi \geq 4$. Moreover, in the case where $\chi>4$, there are two steady states, $\left(\mu_{-} e^{-\chi \mu_{-}^{2} x}, \mu_{-}\right)$and $\left(\mu_{+} e^{-\chi \mu_{+}^{2} x}, \mu_{+}\right)$with $0<\mu_{-}<\mu_{+}<1$.

Proof. The only part to prove is that any steady state has identical values for the two cells. A straightforward computation yields that $\left(\bar{n}_{i}, \bar{\mu}_{i}\right)_{i=1,2}$ satisfies, for $i=1$ and 2 , the following system:

$$
\left\{\begin{aligned}
\bar{n}_{i}(x) & =\overline{\mu_{i}} \exp \left(-\chi \bar{\mu}_{1} \bar{\mu}_{2} x\right), \quad x \geq 0 \\
0 & =\chi \bar{\mu}_{1} \bar{\mu}_{2}-\chi \bar{\mu}_{i}+1
\end{aligned}\right.
$$

From the second equation in the previous system it follows that $\bar{\mu}_{1}=\bar{\mu}_{2}$, hence it yields to 39 which admits a real solution $\bar{\mu}$ iff $\chi \geq 4$.

Lemma A.2. Assume that $\chi>4$, and let $\bar{\mu} \in\left\{\mu_{-}, \mu_{+}\right\}$. The linearized system associated to (2)-(3)-(4) with (5) does not admit 0 as an eigenvalue. Moreover, a complex $\lambda \neq 0$ satisfying $\Re(\lambda) \geq 0$ is an eigenvalue of the linear system if and only if it satisifies

$$
\left(\chi \bar{\mu}^{2}+\beta_{1}\right)\left(\lambda+2 \frac{\chi^{2} \bar{\mu}^{4}}{\lambda}+1\right)+2 \chi \bar{\mu}^{2}-\lambda=0
$$

where $\beta_{1}$ is the unique root of the equation $X^{2}+\chi \bar{\mu}^{2} X-\lambda=0$ satisfying $\Re\left(\beta_{1}\right)<-\chi \bar{\mu}^{2}$.

Proof of Lemma A.2. Let us consider zero mass perturbations around the steady state $(\bar{n}, \bar{\mu})$ solution to $(39)$. For $i=1$ or 2 , we seek the first order modes as follows

$$
\left\{\begin{aligned}
n_{i}(t, x) & =\bar{n}(x)+\widetilde{n}_{i}(x) \exp (\lambda t)+\text { higher order terms, } \quad x \geq 0 \\
\mu_{i}(t) & =\bar{\mu}+\widetilde{\mu}_{i} \exp (\lambda t)+\text { higher order terms } \\
0 & =\int_{0}^{+\infty} \widetilde{n}_{i}(t, x) \mathrm{d} x+\widetilde{\mu}_{i}
\end{aligned}\right.
$$


where $\lambda \in \mathbb{C}$ is some eigenvalue. We linearize (2)-(3)-(4) and we obtain two systems for $i=1,2$ :

$$
\left\{\begin{aligned}
\lambda \widetilde{n}_{i}(x) & =\widetilde{n}_{i}^{\prime \prime}(x)+\chi \bar{\mu}^{2} \widetilde{n}_{i}^{\prime}(x)-\chi^{2} \bar{\mu}^{4} \exp \left(-\chi \bar{\mu}^{2} x\right)\left(\widetilde{\mu}_{1}+\widetilde{\mu}_{2}\right) \\
\lambda \widetilde{\mu}_{i} & =\widetilde{n}_{i}(0)-\widetilde{\mu}_{i} \\
\lambda \widetilde{\mu}_{i} & =\widetilde{n}_{i}^{\prime}(0)+\chi \bar{\mu}^{2}\left(\widetilde{n}_{i}(0)+\widetilde{\mu}_{1}+\widetilde{\mu}_{2}\right)
\end{aligned}\right.
$$

We first investigate the condition for having $\Re(\lambda) \geq 0$ (linear instability). In the case where $\chi>4$, the roots of $X^{2}+\chi \bar{\mu}^{2} X-\lambda=0$ are

$$
\beta_{1}=\frac{-\chi \bar{\mu}^{2}-\sqrt{\chi^{2} \bar{\mu}^{4}+4 \lambda}}{2} \text { and } \quad \beta_{2}=\frac{-\chi \bar{\mu}^{2}+\sqrt{\chi^{2} \bar{\mu}^{4}+4 \lambda}}{2}
$$

Here we have abusively denoted by $\sqrt{\chi^{2} \bar{\mu}^{4}+4 \lambda}$ the only complex number with positive real part satisfying $z^{2}=\chi^{2} \bar{\mu}^{4}+4 \lambda$. With these notations, we have $\Re\left(\beta_{1}\right) \leq-\chi \bar{\mu}^{2}, \Re\left(\beta_{2}\right) \geq 0$, the inequalities being strict as soon as we have $\lambda \neq 0$. Hence, the solution to 42 can be written as:

$$
\begin{cases}\widetilde{n}_{i}(x)=C_{i} \exp \left(\beta_{1} x\right)+D_{i} \exp \left(\beta_{2} x\right)-\frac{\chi^{2} \bar{\mu}^{4}}{\lambda} \exp \left(-\chi \bar{\mu}^{2} x\right)\left(\widetilde{\mu}_{1}+\widetilde{\mu}_{2}\right), & \text { if } \lambda \neq 0 \\ \widetilde{n}_{i}(x)=C_{i} \exp \left(-\chi \bar{\mu}^{2} x\right)+D_{i}-\chi \bar{\mu}^{2} x \exp \left(-\chi \bar{\mu}^{2} x\right)\left(\widetilde{\mu}_{1}+\widetilde{\mu}_{2}\right), & \text { if } \lambda=0\end{cases}
$$

The perturbations must be integrable, so that $D_{i}=0$.

We first establish that $\lambda=0$ cannot be an eigenvalue. Indeed from 42 we deduce that $C_{i}=\widetilde{\mu}_{i}$. Then, the zero integrability condition in (41) yields that $\widetilde{\mu}_{i}=\chi \bar{\mu}^{2} \widetilde{\mu}_{j}, i \neq j$, and thus $\chi^{2} \bar{\mu}^{4}=1$ if $\widetilde{\mu}_{i} \neq 0$. This can only happen if $\chi=4$ and $\bar{\mu}=\frac{1}{2}$. Consequently 0 is not an eigenvalue for the linearized system for $\chi>4$. From now on, we can assume that

$$
\Re(\lambda) \geq 0, \quad \lambda \neq 0, \quad \Re\left(\beta_{1}\right)<-\chi \bar{\mu}^{2} .
$$

The last two equations of 42 now read as the two systems

$$
\left\{\begin{array}{l}
(\lambda+1) \widetilde{\mu}_{i}-C_{i}=-\frac{\chi^{2} \bar{\mu}^{4}}{\lambda}\left(\widetilde{\mu}_{1}+\widetilde{\mu}_{2}\right), \\
\lambda \widetilde{\mu}_{i}-\left(\chi \bar{\mu}^{2}+\beta_{1}\right) C_{i}=\chi \bar{\mu}^{2}\left(\widetilde{\mu}_{1}+\widetilde{\mu}_{2}\right) .
\end{array} \quad, \quad i=1,2 .\right.
$$

Since $\chi \bar{\mu}^{2} \beta_{1}+\beta_{1}^{2}=\lambda$, we notice that

$$
\operatorname{det}\left(\begin{array}{cc}
\lambda+1 & -1 \\
\lambda & -\left(\chi \bar{\mu}^{2}+\beta_{1}\right)
\end{array}\right)=\underbrace{\left(\chi \bar{\mu}^{2}+\beta_{1}\right)}_{\neq 0} \underbrace{\left(-\lambda-1+\beta_{1}\right)}_{\Re(\cdot)<-\chi \bar{\mu}^{2}-1} \neq 0 .
$$

This provides the additional informations $C_{1}=C_{2}=C$ and $\widetilde{\mu}_{1}=\widetilde{\mu}_{2}=\widetilde{\mu}$. Writing back system (43) in terms of $C, \mu$ only, the existence of a nonzero solution to 43 is then equivalent to the degeneracy of the obtained system i.e. 40 is fullfilled.

Lemma A.3. There exists an eigenvalue with nonnegative real part $\Re(\lambda) \geq 0$ if and only if $\chi \bar{\mu}^{2} \leq 1$.

Proof of lemmaA.3. We argue by contradiction. Denoting by $x=\frac{\lambda}{\chi^{2} \bar{\mu}^{4}}$ and $\gamma=\chi \bar{\mu}^{2}$ and recalling the expression of $\beta_{1}$, equation 40 is equivalent to the following one:

$$
\frac{\gamma}{2}\left((1-\sqrt{1+4 x})\left(\gamma^{2} x+\frac{2}{x}+1\right)+4-2 \gamma x\right)=0 .
$$


It is convenient to introduce $2 z=\sqrt{1+4 x}-1$, such that $4 x=(2 z+1)^{2}-1=4 z(z+1)$. Notice that $\lambda$ has a nonnegative real part $\Re(\lambda) \geq 0$ if and only if $z$ has a nonnegative real part $\Re(z) \geq 0$. The equation (44) is equivalent to the following series of equations, since $z \neq 0$ :

$$
\begin{array}{r}
-2 z\left(\gamma^{2} z(z+1)+\frac{2}{z(z+1)}+1\right)+4-2 \gamma z(z+1)=0 \\
-2 \gamma^{2} z^{2}(z+1)+\frac{4 z}{(z+1)}-2 z-2 \gamma z(z+1)=0 \\
-\gamma^{2} z(z+1)^{2}+2-(z+1)-\gamma(z+1)^{2}=0 \\
\gamma^{2} z^{3}+\gamma(2 \gamma+1) z^{2}+(\gamma+1)^{2} z+\gamma-1=0 .
\end{array}
$$

By monotonicity, the latter cubic polynomial has a nonnegative real root if and only if $\gamma \leq 1$, i.e. $\chi \bar{\mu}^{2} \leq 1$. In the case $\gamma>1$, it cannot have a complex root $\omega$ such that $\Re(\omega) \geq 0$ either. Suppose it is the case. Then, $\bar{\omega}$ is another root, and the relations between roots and coeffients imply that

$$
-\gamma^{2}(2 \Re(\omega)+\xi)=\gamma(2 \gamma+1) \Longleftrightarrow 2 \Re(\omega)=-\xi-2-\frac{1}{\gamma},
$$

where $\xi$ is the real root of the cubic polynomial. To conclude, it is enough to show that $\xi>-2$. Indeed, by evaluating the cubic polynomial at $z=-2$, we obtain:

$$
-8 \gamma^{2}+4 \gamma(2 \gamma+1)-2(\gamma+1)^{2}+\gamma-1=-2 \gamma^{2}+\gamma-3<0
$$

This concludes the proof of Lemma A.3.

The stability results stated in Proposition 1.1 follows from Lemma A.1 and Lemma A.3, and the following observation about the roots of $P$ :

$$
\gamma_{-}=\chi \mu_{-}^{2}<1<\gamma_{+}=\chi \mu_{+}^{2} .
$$

\section{Appendix B. Definitions And useful inequalities}

Let us recall some classical definitions. Given two density probabilities $p, q$ on $\mathbb{R}_{+}$, we will in the sequel makes the abuse of notation denoting $p$ and $q$ the corresponding probability measures on $\mathbb{R}_{+}$.

Definition B.1. We define the relative entropy of $p$ with respect to $q$ by

$$
\mathcal{H}(p \mid q)=\int_{0}^{\infty} p(x) \log \frac{p(x)}{q(x)} \mathrm{d} x=\int_{0}^{\infty} q(x)\left(\frac{p(x)}{q(x)} \log \frac{p(x)}{q(x)}-\frac{p(x)}{q(x)}+1\right) \mathrm{d} x \geq 0 .
$$

The Fisher information of $p$ with respect to $q$ is defined as the quantity

$$
\mathcal{I}(p \mid q)=\int_{0}^{\infty} p(x)\left(\partial_{x} \log \frac{p(x)}{q(x)}\right)^{2} \mathrm{~d} x .
$$

Moreover if $p, q$ have finite second moment, 37], the quadratic Wasserstein distance $W(p, q)$ is defined by

$$
W(p, q)=\sqrt{\inf _{\pi \in \Pi(p, q)} \iint_{\mathbb{R}_{+} \times \mathbb{R}_{+}}|x-y|^{2} \mathrm{~d} \pi(x, y)},
$$

where $\Pi(p, q)$ denotes the set of probability measures on $\mathbb{R}_{+} \times \mathbb{R}_{+}$with marginals $p$ and $q$. 
Finally, we consider similarly another Wasserstein distance

$$
W_{1}(p, q)=\inf _{\pi \in \Pi(p, q)} \iint_{\mathbb{R}_{+} \times \mathbb{R}_{+}}|x-y| \mathrm{d} \pi(x, y),
$$

There are several results concerning possible representations of Wasserstein distances when it is specialized to the real line. In such a case it can be considerably simplified in terms of the distribution functions $F(x)=$ $\int_{0}^{x} p(y) \mathrm{d} y, x \in(0, \infty)$, associated to probability measures $p, 37$.

Theorem B.2 (Representation for $W$ ). Let $p$ and $q$ be probability measures on $\mathbb{R}_{+}$with respective distribution functions $F$ and $G$. Then

and

$$
W^{2}(p, q)=\int_{0}^{1}\left|F^{-1}(t)-G^{-1}(t)\right|^{2} \mathrm{~d} t
$$

$$
W_{1}(p, q)=\int_{0}^{1}\left|F^{-1}(t)-G^{-1}(t)\right| \mathrm{d} t=\int_{0}^{\infty}|F(x)-G(x)| \mathrm{d} x,
$$

where $F^{-1}$ is the pseudo-inverse function:

$$
F^{-1}(t)=\inf \{x \in \mathbb{R}: F(x) \geq t\}, 0<t<1 .
$$

Lemma B.3. Let $(\bar{p}, \underline{p})$, and $(n, m)$ be two pairs of probability measures on $\mathbb{R}_{+}$with distribution functions $(\bar{P}, \underline{P})$ and $(N, M)$, respectively. Suppose that they are ordered such that $\underline{P} \leq N, M \leq \bar{P}$, then the Wasserstein distances are ordered as follows,

$$
W(n, m) \leq W(\underline{p}, \bar{p}), \quad W_{1}(n, m) \leq W_{1}(\underline{p}, \bar{p}) .
$$

The following lemma can be applied to interpolation between $p$ and $\delta_{0}$.

Lemma B.4. Let $p, q$ be two probability measures on $\mathbb{R}_{+}$with finite $r$ moment for $r \in\{1,2\}$ and let $0<\mu<1$, then

$$
W_{r}\left(\mu \delta_{0}+(1-\mu) p, \mu \delta_{0}+(1-\mu) q\right)=(1-\mu)^{1 / r} W(p, q) .
$$

Proof. This results is of course valid for any $r \geq 1$. It can be established by direct computation on the pseudo inverse.

The two following inequalities are useful to link the relative entropy, and the Fisher information arising as a part of the entropy dissipation, see [37.

Lemma B.5 (Log-Sobolev inequality). Assume that $q$ satisfies a Gaussian concentration principle i.e. $q(x)=$ $e^{-V(x)}$ with $V^{\prime \prime}(x) \geq \alpha>0$, then the logarithmic Sobolev inequality holds true

$$
\mathcal{I}(p \mid q) \geq 2 \alpha \mathcal{H}(p \mid q) .
$$

Lemma B.6 (HWI inequality for exponential measure). Assume that $q(x)=\lambda e^{-\lambda x}$ then the following inequality holds true

$$
\mathcal{I}(p \mid q) \geq\left(\frac{\mathcal{H}(p \mid q)}{W(p, q)}\right)^{2}
$$

Usually, convergence in the $L^{1}$ norm is controlled by the entropy by means of the Csiszár-Kullback inequality, [15.25].

Proposition B.7 (Csiszár-Kullback inequality). For any non-negative functions $f, g \in L^{1}\left(\mathbb{R}_{+}\right)$such that $\int_{\mathbb{R}_{+}} f(x) \mathrm{d} x=\int_{\mathbb{R}_{+}} g(x) \mathrm{d} x=M$, we have that

$$
\|f-g\|_{1}^{2} \leq 2 M \int_{0}^{\infty} f(x) \log \left(\frac{f(x)}{g(x)}\right) \mathrm{d} x .
$$




\section{Appendix C. Well-posedness of the Coupled PDE-ODE system}

Well-posedness of the Cauchy problem (22)-(3)-(4) relies on a fixed point theorem, as usual. For the sake of clarity, we restrict to a single coupling between the cell bulk density $n(t, x)$ and the boundary value $\mu(t)$. It contains the principal technical difficulty which arises from the coupling between the ODE for $\mu$ and the PDE for $n$. We believe that the extension to the case of pairwise interactions between two cells is straightforward based on the estimates derived below.

We proceed into two steps. Firstly, we obtain refined entropy estimates for the sole PDE problem, without the coupling. As such, we consider two given functions $\mu_{1}(t)$ and $\mu_{2}(t)$, and we derive suitable contraction estimates on $n_{1}, n_{2}$. We introduce the coupling in a second step.

\section{C.1. Entropy estimates for the uncoupled PDE}

Given the function $\mu$, and a smooth nonnegative function $\chi$, we consider the solution of the problem

$$
\left\{\begin{array}{l}
\partial_{t} n(t, x)-\partial_{x x} n(t, x)-\chi(\mu(t)) \partial_{x} n(t, x)=0, \quad x>0 \\
\partial_{x} n(t, 0)+\chi(\mu(t)) n(t, 0)=n(t, 0)-\mu(t), \\
n(0, x)=n^{0}(x), \quad x>0
\end{array}\right.
$$

It is classical that if we consider $\mu \geq 0$ and nonnegative initial data $n^{0}$, then there exists a unique solution which is also nonnegative. Moreover the following estimate

$$
\frac{d}{d t} \int_{0}^{\infty}(1+x) n d x=\mu(t)-\chi(\mu(t)) \int_{0}^{\infty} n d x
$$

ensures that $\int_{0}^{\infty}(1+x) n d x \leq \int_{0}^{\infty}(1+x) n^{0} d x+\int_{0}^{t} \mu d t$. Also, the classical entropy estimate

$$
\frac{d}{d t} \int_{0}^{\infty}(n \log n-n) d x=
$$

In order to compare two solutions $n_{1}, n_{2}$ associated with two inputs $\mu_{1}, \mu_{2}$, we introduce the following Gajewski metric 20]:

$$
d_{G}\left(n_{1}, n_{2}\right)=\int_{0}^{\infty} \underbrace{H\left(n_{1}\right)+H\left(n_{2}\right)-2 H\left(\frac{n_{1}+n_{2}}{2}\right) d x}_{\Delta_{H}\left(n_{1}, n_{2}\right) \geq 0},
$$

where $H$ is the convex function $H(a)=a \log a-a+1$. In particular, for all $a, b$, we have:

$$
H(b)-H\left(\frac{a+b}{2}\right)=H^{\prime}\left(\frac{a+b}{2}\right)\left(\frac{b-a}{2}\right)+\frac{1}{2} H^{\prime \prime}(c)\left(\frac{b-a}{2}\right)^{2},
$$

for some $c \in[a, b]$, and a similar estimate at point $a$. This yields

$$
\Delta_{H}(a, b)=H(a)+H(b)-2 H\left(\frac{a+b}{2}\right) \geq \frac{1}{4} \frac{(b-a)^{2}}{\max (a, b)} \geq \frac{1}{4} \frac{(b-a)^{2}}{a+b},
$$

and similarly

$$
\Delta_{H}(a, b) \leq \frac{1}{4} \frac{(b-a)^{2}}{\min (a, b)}
$$


This pointwise inequality turns into the following integral inequality by Cauchy-Scwhwarz:

$$
d_{G}\left(n_{1}, n_{2}\right) \geq \frac{1}{4} \int_{0}^{\infty} \frac{\left(n_{1}-n_{2}\right)^{2}}{n_{1}+n_{2}} d x \geq \frac{1}{4}\left(\int_{0}^{\infty} n_{1} d x+\int_{0}^{\infty} n_{2} d x\right)^{-1}\left(\int_{0}^{\infty} n_{1} d x-\int_{0}^{\infty} n_{2} d x\right)^{2} .
$$

On the other hand, we have

$$
\begin{aligned}
\frac{d}{d t}\left(d_{G}\left(n_{1}, n_{2}\right)\right) & =\int_{0}^{\infty} \log \left(\frac{2 n_{1}}{n_{1}+n_{2}}\right) \partial_{t} n_{1}+\log \left(\frac{2 n_{2}}{n_{1}+n_{2}}\right) \partial_{t} n_{2} \\
& =-\log \left(\frac{2 n_{1}(0)}{n_{1}(0)+n_{2}(0)}\right)\left(n_{1}(0)-\mu_{1}\right)-\log \left(\frac{2 n_{2}(0)}{n_{1}(0)+n_{2}(0)}\right)\left(n_{2}(0)-\mu_{2}\right) \\
& -\int_{0}^{\infty} \partial_{x} \log \left(\frac{2 n_{1}}{n_{1}+n_{2}}\right)\left(\partial_{x} n_{1}+\chi_{1} n_{1}\right)-\int_{0}^{\infty} \partial_{x} \log \left(\frac{2 n_{2}}{n_{1}+n_{2}}\right)\left(\partial_{x} n_{2}+\chi_{2} n_{2}\right)
\end{aligned}
$$

In the sequel, we use the notation $q_{i}=\frac{2 n_{i}}{n_{1}+n_{2}}$ and notice that $q_{1}+q_{2}=2$ and thus $\partial_{x} q_{1}=-\partial_{x} q_{2}$. The previous estimate is equivalent to the following one:

$$
\begin{aligned}
\frac{d}{d t} d_{G}\left(n_{1}, n_{2}\right) & =\mu_{1} \log q_{1}(0)+\mu_{2} \log q_{2}(0)-\Delta_{H}\left(n_{1}(0), n_{2}(0)\right) \\
& -\int_{0}^{\infty}\left(\frac{n_{1}+n_{2}}{2}\right) \partial_{x} q_{1}\left(\partial_{x} \log n_{1}+\chi_{1}\right)-\int_{0}^{\infty}\left(\frac{n_{1}+n_{2}}{2}\right) \partial_{x} q_{2}\left(\partial_{x} \log n_{2}+\chi_{2}\right) .
\end{aligned}
$$

To bound the cross term involving $\mu_{i}$ and $n_{i}(0)$ in the first line, we notice that $\mu_{1} \log q+\mu_{2} \log (2-q)$ reaches a maximum for the critical value $q^{*}$ satisfying

$$
\frac{\mu_{1}}{q^{*}}=\frac{\mu_{2}}{2-q^{*}}, \quad q^{*}=\frac{2 \mu_{1}}{\mu_{1}+\mu_{2}} .
$$

Therefore, the first line is upper bounded by

$$
\Delta_{H}\left(\mu_{1}, \mu_{2}\right)=\mu_{1} \log \left(\frac{2 \mu_{1}}{\mu_{1}+\mu_{2}}\right)+\mu_{2} \log \left(\frac{2 \mu_{2}}{\mu_{1}+\mu_{2}}\right)
$$

We arrive at

$$
\begin{aligned}
\frac{d}{d t} d_{G}\left(n_{1}, n_{2}\right) & \leq \Delta_{H}\left(\mu_{1}, \mu_{2}\right)-\Delta_{H}\left(n_{1}(0), n_{2}(0)\right) \\
& -\int_{0}^{\infty}\left(\frac{n_{1}+n_{2}}{2}\right) \partial_{x} q_{1}\left(\partial_{x} \log n_{1}+\chi_{1}\right)-\int_{0}^{\infty}\left(\frac{n_{1}+n_{2}}{2}\right) \partial_{x} q_{2}\left(\partial_{x} \log n_{2}+\chi_{2}\right) \\
& =\Delta_{H}\left(\mu_{1}, \mu_{2}\right)-\Delta_{H}\left(n_{1}(0), n_{2}(0)\right) \\
& -\int_{0}^{\infty}\left(\frac{n_{1}+n_{2}}{2}\right) \partial_{x} q_{1}\left(\partial_{x} \log \left(\frac{n_{1}}{n_{2}}\right)+\chi_{1}-\chi_{2}\right) .
\end{aligned}
$$

We recall that $\frac{n_{1}}{n_{2}}=\frac{q_{1}}{2-q_{1}}$, so that $\partial_{x} \log \frac{n_{1}}{n_{2}}=\partial_{x} q_{1}\left(\frac{1}{q_{1}}+\frac{1}{2-q_{1}}\right)$. Hence we obtain

$$
\begin{aligned}
\frac{d}{d t} d_{G}\left(n_{1}, n_{2}\right) & \leq \Delta_{H}\left(\mu_{1}, \mu_{2}\right)-\Delta_{H}\left(n_{1}(0), n_{2}(0)\right) \\
& -\int_{0}^{\infty}\left(\frac{n_{1}+n_{2}}{2}\right)\left(\frac{1}{q_{1}}+\frac{1}{2-q_{1}}\right)\left|\partial_{x} q_{1}\right|^{2}-\left(\chi_{1}-\chi_{2}\right) \int_{0}^{\infty}\left(\frac{n_{1}+n_{2}}{2}\right) \partial_{x} q_{1}
\end{aligned}
$$


Using again the relationship $q_{1}+q_{2}=2$, we can rewrite the last but one integral term as

$$
\begin{aligned}
\int_{0}^{\infty}\left(\frac{n_{1}+n_{2}}{2}\right)\left(\frac{1}{q_{1}}+\frac{1}{2-q_{1}}\right)\left|\partial_{x} q_{1}\right|^{2} & =\int_{0}^{\infty}\left(\frac{n_{1}+n_{2}}{2}\right)\left(q_{1}\left|\partial_{x} \log q_{1}\right|^{2}+q_{2}\left|\partial_{x} \log q_{2}\right|^{2}\right) \\
& =\int_{0}^{\infty} n_{1}\left|\partial_{x} \log q_{1}\right|^{2}+\int_{0}^{\infty} n_{2}\left|\partial_{x} \log q_{2}\right|^{2} .
\end{aligned}
$$

In addition, we notice that

$$
\begin{aligned}
\left|\left(\chi_{1}-\chi_{2}\right) \int_{0}^{\infty}\left(\frac{n_{1}+n_{2}}{2}\right) \partial_{x} q_{1}\right| & \leq\left|\chi_{1}-\chi_{2}\right| \int_{0}^{\infty}\left(\frac{n_{1}+n_{2}}{2}\right)\left|q_{1}\right|\left|\partial_{x} \log q_{1}\right| \\
& \leq \frac{\left|\chi_{1}-\chi_{2}\right|^{2}}{2} \int_{0}^{\infty} n_{1}+\frac{1}{2} \int_{0}^{\infty} n_{1}\left|\partial_{x} \log q_{1}\right|^{2}
\end{aligned}
$$

Combining the integral terms, and symmetrizing the roles of $n_{1}, n_{2}$, we end up with our key estimate:

$$
\begin{aligned}
\frac{d}{d t} d_{G}\left(n_{1}, n_{2}\right) \leq \Delta_{H}\left(\mu_{1}, \mu_{2}\right)-\Delta_{H}\left(n_{1}(0), n_{2}(0)\right)+\frac{\left|\chi_{1}-\chi_{2}\right|^{2}}{2} & \int_{0}^{\infty} \frac{n_{1}+n_{2}}{2} \\
& -\frac{3}{4}\left(\int_{0}^{\infty} n_{1}\left|\partial_{x} \log q_{1}\right|^{2}+\int_{0}^{\infty} n_{2}\left|\partial_{x} \log q_{2}\right|^{2}\right)
\end{aligned}
$$

\section{C.2. The fixed point mapping}

For a given function $\mu(t)$, we define $n(t, x)$ as in Section C.1. Next, we define $\mu^{\text {out }}(t)$ as a solution of the following ODE:

$$
\frac{d}{d t} \mu^{\text {out }}(t)=n(t, 0)-\mu^{\text {out }}(t), \quad \text { or equivalently } \quad \mu^{\text {out }}(t)=e^{-t} \mu^{\text {out }}(0)+\int_{0}^{t} e^{s-t} n(s, 0) d s
$$

with the initial data $\mu^{\text {out }}(0)=1-\int_{0}^{\infty} n^{0}(x) d x>0$. On the other hand, $n(t, x)$ and $\mu(t)$ satisfy the following relationship, obtained by integrating (45) with respect to space:

$$
\frac{d}{d t} \int_{0}^{\infty} n(t, x) d x=\mu(t)-n(t, 0)
$$

Consequently, 52 can be recast as:

$$
\mu^{\text {out }}(t)=e^{-t} \mu^{\text {out }}(0)+\int_{0}^{t} e^{s-t} \mu(s) d s+\int_{0}^{t} e^{s-t} I(s) d s-I(t)+e^{-t} I(0), \quad I(t)=\int_{0}^{\infty} n(t, x) d x .
$$

Suppose we are given two input functions $\mu_{i}, i=1,2$, then the two output functions $\mu_{i}^{\text {out }}, i=1,2$ share the same initial data. Moreover, denoting $I_{i}=\int_{0}^{\infty} n_{i}(t, x) d x$, we have

$$
\mu_{1}^{\text {out }}(t)-\mu_{2}^{\text {out }}(t)=I_{2}(t)-I_{1}(t)+\int_{0}^{t} e^{s-t}\left(I_{1}(s)-I_{2}(s)\right) d s+\int_{0}^{t} e^{s-t}\left(\mu_{1}(s)-\mu_{2}(s)\right) d s
$$

We deduce from our key estimate (51) that

$$
d_{G}\left(n_{1}(t), n_{2}(t)\right) \leq \int_{0}^{t} \Delta_{H}\left(\mu_{1}(s), \mu_{2}(s)\right) d s+\frac{1}{4}(\operatorname{Lip} \chi)^{2} \int_{0}^{t}\left|\mu_{1}(s)-\mu_{2}(s)\right|^{2}\left(I_{1}(s)+I_{2}(s)\right) d s,
$$


where $\operatorname{Lip} \chi=\sup _{\mu \in(0,1)}\left|\chi^{\prime}(\mu)\right|$. Finally, using the two pointwise inequalities 447)-48,, we obtain

$$
\frac{\left(I_{1}(t)-I_{2}(t)\right)^{2}}{\max \left(I_{1}(t), I_{2}(t)\right)} \leq \int_{0}^{t} \frac{\left(\mu_{1}(s)-\mu_{2}(s)\right)^{2}}{\min \left(\mu_{1}(s), \mu_{2}(s)\right)} d s+(\operatorname{Lip} \chi)^{2} \int_{0}^{t}\left|\mu_{1}(s)-\mu_{2}(s)\right|^{2}\left(I_{1}(s)+I_{2}(s)\right) d s .
$$

By the combination of (55) and (57), we obtain a contraction estimate of the form

$$
\sup _{t \in[0, T]}\left|\mu_{1}^{\text {out }}(t)-\mu_{2}^{\text {out }}(t)\right| \leq \omega(T) \sup _{t \in(0, T)}\left|\mu_{1}(t)-\mu_{2}(t)\right|,
$$

where $\omega(T)=\mathcal{O}(\sqrt{T})$, provided that $\mu_{i}, i=1,2$ are uniformly bounded below, and $I_{i}, i=1,2$ are uniformly bounded above on $[0, T]$. The latter condition is clearly verified on the appropriate functional space:

$$
X_{T}=\left\{\mu \in C([0, T]), \mu(0)=1-\int_{0}^{\infty} n^{0}(x) d x, \quad \mu(0) e^{-t} \leq \mu(t) \leq 1\right\}
$$

at least for short time, depending on $n^{0}(x)$. It remains to check that the solution $n(t, x)$ of the coupled problem is such that $\mu(t)$ stays uniformly strictly above 0 and below 1 on $[0, T]$ for arbitrary time $T$. The former is clear from the estimate $\mu(0) e^{-t} \leq \mu(t)$. The latter can be derived using the conservation relation $\mu(t)+\int_{0}^{\infty} n d x=1$, and the fact that $n$ cannot vanish in finite time. 